\title{
Space-time computational analysis of tire aerodynamics with actual geometry, road contact, tire deformation, road roughness and fluid film
}

\author{
Takashi Kuraishi $^{1} \cdot$ Kenji Takizawa $^{1} \cdot$ Tayfun E. Tezduyar $^{2,3}$
}

Received: 31 March 2019 / Accepted: 15 June 2019 / Published online: 2 July 2019

(c) The Author(s) 2019

\begin{abstract}
The space-time (ST) computational method "ST-SI-TC-IGA" has recently enabled computational analysis of tire aerodynamics with actual tire geometry, road contact and tire deformation. The core component of the ST-SI-TC-IGA is the ST Variational Multiscale (ST-VMS) method, and the other key components are the ST Slip Interface (ST-SI) and ST Topology Change (ST-TC) methods and the ST Isogeometric Analysis (ST-IGA). These ST methods played their parts in overcoming the computational challenges, including (i) the complexity of an actual tire geometry with longitudinal and transverse grooves, (ii) the spin of the tire, (iii) maintaining accurate representation of the boundary layers near the tire while being able to deal with the flow-domain topology change created by the road contact, and (iv) the turbulent nature of the flow. The combination of the ST-VMS, ST-SI and the ST-IGA has also recently enabled solution of fluid film problems with a computational cost comparable to that of the Reynolds-equation model for the comparable solution quality. This was accomplished with the computational flexibility to go beyond the limitations of the Reynolds-equation model. Here we include and address the computational challenges associated with the road roughness and the fluid film between the tire and the road. The new methods we add to accomplish that include a remedy for the trapped fluid, a method for reducing the number of control points as a space occupied by the fluid shrinks down to a narrow gap, and a method for representing the road roughness. We present computations for a 2D test problem with a straight channel, a simple 2D model of the tire, and a 3D model with actual tire geometry and road roughness. The computations show the effectiveness of our integrated set of ST methods targeting tire aerodynamics.
\end{abstract}

Keywords Tire aerodynamics · Actual tire geometry $\cdot$ Road contact $\cdot$ Road roughness $\cdot$ Fluid film $\cdot$ ST Variational Multiscale (ST-VMS) method · ST Slip Interface (ST-SI) method $\cdot$ ST Topology Change (ST-TC) method $\cdot$ ST Isogeometric Analysis (ST-IGA)

\section{Introduction}

Computational analysis of tire aerodynamics with actual tire geometry, road contact and tire deformation poses tough

Kenji Takizawa

Kenji.Takizawa@tafsm.org

Tayfun E. Tezduyar

tezduyar@tafsm.org

1 Department of Modern Mechanical Engineering, Waseda University, 3-4-1 Ookubo, Shinjuku-ku, Tokyo 169-8555, Japan

2 Mechanical Engineering, Rice University - MS 321, 6100 Main Street, Houston, TX 77005, USA

3 Faculty of Science and Engineering, Waseda University, 3-4-1 Ookubo, Shinjuku-ku, Tokyo 169-8555, Japan challenges [1]. The challenges include (i) the complexity of an actual tire geometry with longitudinal and transverse grooves, (ii) the spin of the tire, (iii) maintaining accurate representation of the boundary layers near the tire while being able to deal with the flow-domain topology change created by the road contact, and (iv) the turbulent nature of the flow. These computational challenges were overcome in [1] with the space-time (ST) computational method "ST-SI-TC-IGA" $[1,2]$. The core component of the ST-SI-TC-IGA is the ST Variational Multiscale (ST-VMS) method [3-5], which subsumes its precursor "ST-SUPS" (see Sect. 2). The other key 
components are the ST Slip Interface (ST-SI) $[6,7]$ and ST Topology Change (ST-TC) $[8,9]$ methods and the ST Isogeometric Analysis (ST-IGA) [3,10,11].

The VMS feature of the ST-VMS addresses the challenge created by the turbulent nature of the flow, the moving-mesh feature of the ST framework enables high-resolution flow computation near the moving fluid-solid interfaces, and the higher-order accuracy of the ST framework strengthens both features. The ST-SI enables moving-mesh computation with the tire spinning. The mesh covering the tire spins with it, and the SI between the spinning mesh and the rest of the mesh accurately connects the two sides of the solution. The ST-TC enables moving-mesh computation even with the TC created by the contact between the tire and the road. It deals with the contact while maintaining high-resolution flow representation near the tire. Integration of the ST-SI and ST-TC enables high-resolution representation even though parts of the SI are coinciding with the tire and road surfaces. It also enables dealing with the tire-road contact location change and contact sliding. By integrating the ST-IGA with the ST-SI and ST-TC, in addition to having a more accurate representation of the tire geometry and increased accuracy in the flow solution, the element density in the tire grooves and in the narrow spaces near the contact areas is kept at a reasonable level.

The work report in [1] included computations with the ST-SI-TC-IGA and two models of flow around a rotating tire with road contact and prescribed deformation. One was a simple 2D model for verification purposes, and one was a 3D model with an actual tire geometry and a deformation pattern provided by the tire company. The computations showed the effectiveness of the ST-SI-TC-IGA in tire aerodynamics with actual tire geometry, road contact and tire deformation.

The combination of the ST-VMS, ST-SI and the ST-IGA also created an ST computational method [12] with builtin Reynolds-equation limit, enabling solution of fluid film problems with a computational cost comparable to that of the Reynolds-equation model for the comparable solution quality. This was accomplished with the computational flexibility to go beyond the limitations of the Reynolds-equation model. With the ST-IGA, even with just one quadratic NURBS element across the gap of the fluid film, we reach a solution quality comparable to that of the Reynolds-equation model.

The work reported in [12] included detailed 2D test computations. The computations showed how the ST computational method performs compared to the Reynolds-equation model, and also compared to finite element discretization. The test cases included different circumferential and normal mesh refinement levels. Also included were test cases with an SI in the mesh and cases where the no-slip boundary conditions are enforced weakly.

In this article, we include and address the computational challenges associated with the road roughness and the fluid film between the tire and the road. To accomplish that we add some new methods to the ST computational methods we described in this section so far. The added methods include a remedy for the trapped fluid and a method for reducing the number of control points as a space occupied by the fluid shrinks down to a narrow gap. They also include a method for representing the road roughness. We present computations for a $2 \mathrm{D}$ test problem with a straight channel, a simple 2D model of the tire, and a 3D model with actual tire geometry and road roughness.

In Sect. 2, we provide an overview of the ST-VMS and ST-SUPS. The overviews of the ST-SI, ST-TC, ST-SI-TC, ST-IGA and ST-SI-TC-IGA are provided in Sects. 3-7. The remedy for the trapped fluid is described in Sect. 8. The 2D test computations with a straight channel are presented in Sect. 9. In Sect. 10, we present, in the context of a simple 2D tire model, test computations with different contact representations. The computation with the 3D tire model with actual tire geometry and road roughness is presented in Sect. 11, and the concluding remarks are given in Sect. 12.

\section{ST-VMS and ST-SUPS}

The Deforming-Spatial-Domain/Stabilized ST (DSD/SST) method [13-15] was introduced for computation of flows with moving boundaries and interfaces (MBI), including fluid-structure interaction (FSI). In MBI computations the DSD/SST functions as a moving-mesh method. Moving the fluid mechanics mesh to follow an interface enables mesh-resolution control near the interface and, consequently, high-resolution boundary-layer representation near fluidsolid interfaces. The stabilization components of the original DSD/SST are the Streamline-Upwind/Petrov-Galerkin (SUPG) [16] and Pressure-Stabilizing/Petrov-Galerkin (PSPG) [13] stabilizations, which are used widely. Because of the SUPG and PSPG components, the original DSD/SST is now called "ST-SUPS." The ST-VMS is the VMS version of the DSD/SST. The VMS components of the ST-VMS are from the residual-based VMS (RBVMS) method [1720]. The ST-VMS has two more stabilization terms beyond those in the ST-SUPS, and the additional terms give the method better turbulence modeling features. The ST-SUPS and ST-VMS, because of the higher-order accuracy of the ST framework (see $[3,4]$ ), are desirable also in computations without MBI.

The DSD/SST is an alternative to the Arbitrary Lagrangian-Eulerian (ALE) method, which is an older and more commonly used moving-mesh method. The ALE-VMS method [21-27] is the VMS version of the ALE. It succeeded the ST-SUPS [13] and ALE-SUPS [28] and preceded the ST-VMS. To increase their scope and accuracy, the ALE-VMS and RBVMS are often supplemented with special methods, such as those for weakly-enforced no-slip 
boundary conditions [29-31], "sliding interfaces" [32,33] and backflow stabilization [34]. The ALE-SUPS, RBVMS and ALE-VMS have been applied to many classes of FSI, MBI and fluid mechanics problems. The classes of problems include ram-air parachute FSI [28], wind-turbine aerodynamics and FSI [35-45], more specifically, vertical-axis wind turbines [44-47], floating wind turbines [48], wind turbines in atmospheric boundary layers [43-45,49], and fatigue damage in wind-turbine blades [50], patient-specific cardiovascular fluid mechanics and FSI [21,51-56], biomedicaldevice FSI [57-62], ship hydrodynamics with free-surface flow and fluid-object interaction [63,64], hydrodynamics and FSI of a hydraulic arresting gear [65,66], hydrodynamics of tidal-stream turbines with free-surface flow [67], passive-morphing FSI in turbomachinery [68], bioinspired FSI for marine propulsion [69,70], bridge aerodynamics and fluid-object interaction [71-73], and mixed ALEVMS/Immersogeometric computations [60-62,74,75] in the framework of the Fluid-Solid Interface-Tracking/InterfaceCapturing Technique [76]. Recent advances in stabilized and multiscale methods may be found for stratified incompressible flows in [77], for divergence-conforming discretizations of incompressible flows in [78], and for compressible flows with emphasis on gas-turbine modeling in [79].

The ST-SUPS and ST-VMS have also been applied to many classes of FSI, MBI and fluid mechanics problems (see [80] for a comprehensive summary). The classes of problems include spacecraft parachute analysis for the landing-stage parachutes [24,81-84], cover-separation parachutes [85] and the drogue parachutes [86-88], wind-turbine aerodynamics for horizontal-axis wind-turbine rotors $[24,35,89,90]$, full horizontal-axis wind-turbines [41,91-93] and vertical-axis wind-turbines $[6,44,45]$, flapping-wing aerodynamics for an actual locust $[10,24,94,95]$, bioinspired MAVs [92,93,96,97] and wing-clapping $[8,98]$, blood flow analysis of cerebral aneurysms [92,99], stent-blocked aneurysms [99-101], aortas [102-105] and heart valves [2,8,9,93,104,106,107], spacecraft aerodynamics [85,108], thermo-fluid analysis of ground vehicles and their tires [5,106], thermo-fluid analysis of disk brakes [7], flow-driven string dynamics in turbomachinery [109-111], flow analysis of turbocharger turbines [11,112-114], flow around tires with road contact and deformation [1,106,115,116], fluid films [12], ram-air parachutes [117], and compressible-flow spacecraft parachute aerodynamics [118,119].

The ST-SUPS, ALE-SUPS, RBVMS, ALE-VMS and STVMS all have some embedded stabilization parameters that play a significant role (see [24]). There are many ways of defining these stabilization parameters (for examples, see [1, $5,6,14,15,91,120-136])$. We will specify which ones we use here when we describe the computations in Sects. 9-11.

For more on the ST-VMS and ST-SUPS, see [24]. In the flow analyses presented here, the ST framework pro- vides higher-order accuracy in a general context. The VMS and SUPS features of the ST-VMS and ST-SUPS address the computational challenges associated with the multiscale nature of the unsteady flow. The moving-mesh feature of the ST framework enables high-resolution computation near the tire surfaces.

\section{ST-SI}

The ST-SI was introduced in [6], in the context of incompressible-flow equations, to retain the desirable movingmesh features of the ST-VMS and ST-SUPS when we have spinning solid surfaces, such as a turbine rotor. The mesh covering the spinning surface spins with it, retaining the high-resolution representation of the boundary layers. The starting point in the development of the ST-SI was the version of the ALE-VMS for computations with sliding interfaces $[32,33]$. Interface terms similar to those in the ALE-VMS version are added to the ST-VMS to account for the compatibility conditions for the velocity and stress at the SI. That accurately connects the two sides of the solution. An ST-SI version where the SI is between fluid and solid domains was also presented in [6]. The SI in this case is a "fluid-solid SI" rather than a standard "fluid-fluid SI" and enables weak enforcement of the Dirichlet boundary conditions for the fluid. The ST-SI introduced in [7] for the coupled incompressible-flow and thermal-transport equations retains the high-resolution representation of the thermo-fluid boundary layers near spinning solid surfaces. These ST-SI methods have been applied to aerodynamic analysis of vertical-axis wind turbines [6,44,45], thermo-fluid analysis of disk brakes [7], flow-driven string dynamics in turbomachinery [109-111], flow analysis of turbocharger turbines [11,112-114], flow around tires with road contact and deformation [1,106,115,116], fluid films [12], aerodynamic analysis of ram-air parachutes [117], and flow analysis of heart valves $[2,104,107]$.

In the ST-SI version presented in [6] the SI is between a thin porous structure and the fluid on its two sides. This enables dealing with the porosity in a fashion consistent with how the standard fluid-fluid SIs are dealt with and how the Dirichlet conditions are enforced weakly with fluid-solid SIs. This version also enables handling thin structures that have T-junctions. This method has been applied to incompressibleflow aerodynamic analysis of ram-air parachutes with fabric porosity [117]. The compressible-flow ST-SI methods were introduced in [118], including the version where the SI is between a thin porous structure and the fluid on its two sides. Compressible-flow porosity models were also introduced in [118]. These, together with the compressible-flow ST SUPG method [137], extended the ST computational analysis range to compressible-flow aerodynamics of parachutes with fab- 
ric and geometric porosities. That enabled ST computational flow analysis of the Orion spacecraft drogue parachute in the compressible-flow regime $[118,119]$.

For more on the ST-SI, see [6,7]. In the tire-aerodynamics computational analysis here, the mesh covering the tire spins with it, and the SI between the spinning mesh and the rest of the mesh accurately connects the two sides of the solution. This enables high-resolution representation of the boundary layers near the tire. In the computations here, the ST-SI is used also in combination with the ST-TC, and we will describe the ST-SI-TC in Sect. 5.

\section{ST-TC}

The ST-TC $[8,9]$ was introduced for moving-mesh computation of flow problems with TC, such as contact between solid surfaces. Even before the ST-TC, the ST-SUPS and ST-VMS, when used with robust mesh update methods, have proven effective in flow computations where the solid surfaces are in near contact or create other near TC, if the nearness is sufficiently near for the purpose of solving the problem. Many classes of problems can be solved that way with sufficient accuracy. For examples of such computations, see the references mentioned in [8]. The ST-TC made moving-mesh computations possible even when there is an actual contact between solid surfaces or other TC. By collapsing elements as needed, without changing the connectivity of the "parent" mesh, the ST-TC can handle an actual TC while maintaining high-resolution boundary layer representation near solid surfaces. This enabled successful moving-mesh computation of heart valve flows $[2,8,9,93,104,106,107]$, wing clapping [98], and flow around a rotating tire with road contact and prescribed deformation $[1,106,115,116]$.

For more on the ST-TC, see [8,9]. In the computational analyses here, the ST-TC enables moving-mesh computation even with the TC created by the actual contact between the tire and the road. It deals with the contact while maintaining high-resolution flow representation near the tire.

\section{ST-SI-TC}

The ST-SI-TC is the integration of the ST-SI and ST-TC. A fluid-fluid SI requires elements on both sides of the SI. When part of an SI needs to coincide with a solid surface, which happens for example when the solid surfaces on two sides of an SI come into contact or when an SI reaches a solid surface, the elements between the coinciding SI part and the solid surface need to collapse with the ST-TC mechanism. The collapse switches the SI from fluid-fluid SI to fluidsolid SI. With that, an SI can be a mixture of fluid-fluid and fluid-solid SIs. With the ST-SI-TC, the elements collapse and are reborn independent of the nodes representing a solid surface. The ST-SI-TC enables high-resolution flow representation even when parts of the SI are coinciding with a solid surface. It also enables dealing with contact location change and contact sliding. This was applied to heart valve flow analysis $[2,104,107]$ and tire aerodynamics with road contact and deformation $[1,115,116]$.

For more on the ST-SI-TC, see $[2,115]$. In the computational analyses presented here, the ST-SI-TC enables contact location change and contact sliding between the tire and road surfaces.

\section{ST-IGA}

The ST-IGA is the integration of the ST framework with isogeometric discretization. It was introduced in [3]. Computations with the ST-VMS and ST-IGA were first reported in [3] in a 2D context, with IGA basis functions in space for flow past an airfoil, and in both space and time for the advection equation. Using higher-order basis functions in time enables getting full benefit out of using higher-order basis functions in space. This was demonstrated with the stability and accuracy analysis given in [3] for the advection equation.

The ST-IGA with IGA basis functions in time enables a more accurate representation of the motion of the solid surfaces and a mesh motion consistent with that. This was pointed out in $[3,4]$ and demonstrated in $[10,94,96]$. It also enables more efficient temporal representation of the motion and deformation of the volume meshes, and more efficient remeshing. These motivated the development of the ST/NURBS Mesh Update Method (STNMUM) [10,94,96]; the name was given in [91]. The STNMUM has a wide scope that includes spinning solid surfaces. With the spinning motion represented by quadratic NURBS in time, and with sufficient number of temporal patches for a full rotation, the circular paths are represented exactly. A "secondary mapping" $[3,4,10,24]$ enables also specifying a constant angular velocity for invariant speeds along the circular paths. The ST framework and NURBS in time also enable, with the "ST-C" method, extracting a continuous representation from the computed data and, in large-scale computations, efficient data compression $[5,7,106,109-111,138]$. The STNMUM and the ST-IGA with IGA basis functions in time have been used in many 3D computations. The classes of problems solved are flapping-wing aerodynamics for an actual locust [10,24,94,95], bioinspired MAVs [92,93,96,97] and wingclapping [8,98], separation aerodynamics of spacecraft [85], aerodynamics of horizontal-axis [41,91-93] and verticalaxis $[6,44,45]$ wind-turbines, thermo-fluid analysis of ground vehicles and their tires [5,106], thermo-fluid analysis of disk brakes [7], flow-driven string dynamics in turbomachin- 
ery [109-111], and flow analysis of turbocharger turbines [11,112-114].

The ST-IGA with IGA basis functions in space enables more accurate representation of the geometry and increased accuracy in the flow solution. It accomplishes that with fewer control points, and consequently with larger effective element sizes. That in turn enables using larger time-step sizes while keeping the Courant number at a desirable level for good accuracy. It has been used in ST computational flow analysis of turbocharger turbines [11,112-114], flow-driven string dynamics in turbomachinery [110,111], ram-air parachutes [117], spacecraft parachutes [119], aortas $[104,105]$, heart valves [2,104,107], tires with road contact and deformation [1,116], and fluid films [12]. Using IGA basis functions in space is now a key part of some of the newest arterial zero-stress-state (ZSS) estimation methods [139-142] and related shell analysis [143].

For more on the ST-IGA, see [11,24,94,117]. In the computational flow analyses presented here, the ST-IGA enables more accurate representation of the tire geometry and increased accuracy in the flow solution.

\section{ST-SI-TC-IGA}

The turbocharger turbine analysis [11,112-114] and flowdriven string dynamics in turbomachinery [110,111] were based on the integration of the ST-SI and ST-IGA. The IGA basis functions were used in the spatial discretization of the fluid mechanics equations and also in the temporal representation of the rotor and spinning-mesh motion. That enabled accurate representation of the turbine geometry and rotor motion and increased accuracy in the flow solution. The IGA basis functions were used also in the spatial discretization of the string structural dynamics equations. That enabled increased accuracy in the structural dynamics solution, as well as smoothness in the string shape and fluid dynamics forces computed on the string.

The ram-air parachute analysis [117] and spacecraft parachute compressible-flow analysis [119] were based on the integration of the ST-IGA, the ST-SI version that weakly enforces the Dirichlet conditions, and the ST-SI version that accounts for the porosity of a thin structure. The ST-IGA with IGA basis functions in space enabled, with relatively few number of unknowns, accurate representation of the parafoil and parachute geometries and increased accuracy in the flow solution. The volume mesh needed to be generated both inside and outside the parafoil. Mesh generation inside was challenging near the trailing edge because of the narrowing space. The spacecraft parachute has a very complex geometry, including gores and gaps. Using IGA basis functions addressed those challenges and still kept the ele- ment density near the trailing edge of the parafoil and around the spacecraft parachute at a reasonable level.

The heart valve analysis [2,104,107] was based on the integration of the ST-SI, ST-TC and ST-IGA. The ST-SI-TCIGA, beyond enabling a more accurate representation of the geometry and increased accuracy in the flow solution, kept the element density in the narrow spaces near the contact areas at a reasonable level. When solid surfaces come into contact, the elements between the surface and the SI collapse. Before the elements collapse, the boundaries could be curved and rather complex, and the narrow spaces might have high-aspect-ratio elements. With NURBS elements, it was possible to deal with such adverse conditions rather effectively.

In computational analysis of flow around tires with road contact and deformation [1], the ST-SI-TC-IGA enables a more accurate representation of the geometry and motion of the tire surfaces, a mesh motion consistent with that, and increased accuracy in the flow solution. It also keeps the element density in the tire grooves and in the narrow spaces near the contact areas at a reasonable level. In addition, we benefit from the mesh generation flexibility provided by using SIs.

An SI provides mesh generation flexibility in a general context by accurately connecting the two sides of the solution computed over nonmatching meshes. This type of mesh generation flexibility is especially valuable in complex-geometry flow computations with isogeometric discretization, removing the matching requirement between the NURBS patches without loss of accuracy. This feature was used in the flow analysis of heart valves [2,104,107], turbocharger turbines [11,112-114], and spacecraft parachute compressible-flow analysis [119].

For more on the ST-SI-TC-IGA, see $[1,2]$. In the computations presented here, the ST-SI-TC-IGA is used for the reasons given and as described in the earlier paragraphs of this section.

\section{Remedy for the trapped fluid}

With the ST-SI-TC, we can deal with a TC in the domain, which may create isolated subdomains with trapped fluid. When a subdomain is isolated it may be difficult to satisfy the incompressibility constraint as the volume changes its shape, and that would impact to the solution process. As one way of remedying that, we create a path that enables mass flow between the isolated subdomain and the rest of the domain. To reduce the unintended effects of the path, we make it a narrow gap such that the flow going through it is laminar.

As stated in [12], with a consistent formulation, the Reynolds-equation limit can be represented by a quadratic NURBS element. This means that when the height of the gap is small enough, just one quadratic NURBS element would 
be enough. This motivates us to use a single quadratic element across the gap. As also stated in [12], the quadratic NURBS element would need to have a constant parametrization across the gap. Here we explore how to create that constant-parametrization quadratic NURBS element.

With the ST-SI-TC, we can collapse or re-form space by using a master-slave relationship. With a similar process, we can reduce the number of control points as a space occupied by the fluid shrinks down to a narrow gap. Before describing the process for quadratic NURBS elements, we explain the concept for linear elements in Fig. 1.

While reducing the number of control points is a simple process when we are using linear elements, that is not the case for higher-order NURBS elements. Figure 2 is an example of starting with three quadratic elements and reducing the number of control points. To reduce the number of control points to three, we make two of the control points slaves of the last control point. This results in two elements that are not desirable.
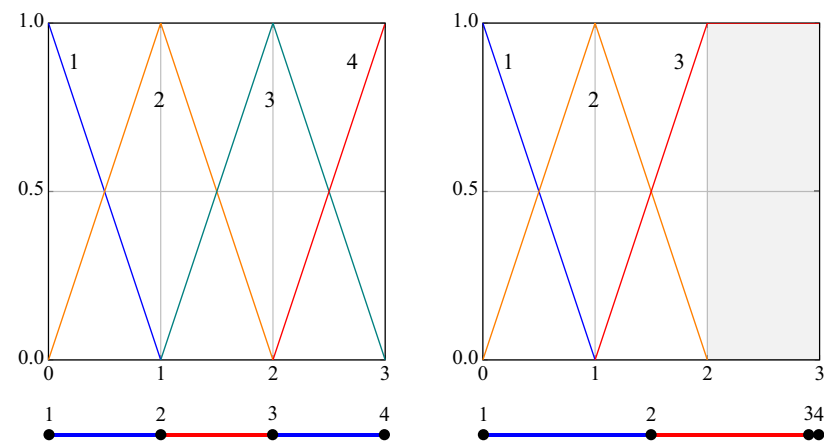

Fig. 1 Meshes and shape functions for the linear elements. The left mesh, which we see as the "parent" mesh, is for a wider stage of the gap. The right mesh is for when the gap is narrow and we reduce the number of control points to three by TC collapse. This results in two elements. The gray zone indicates the unused elements in the parametric space
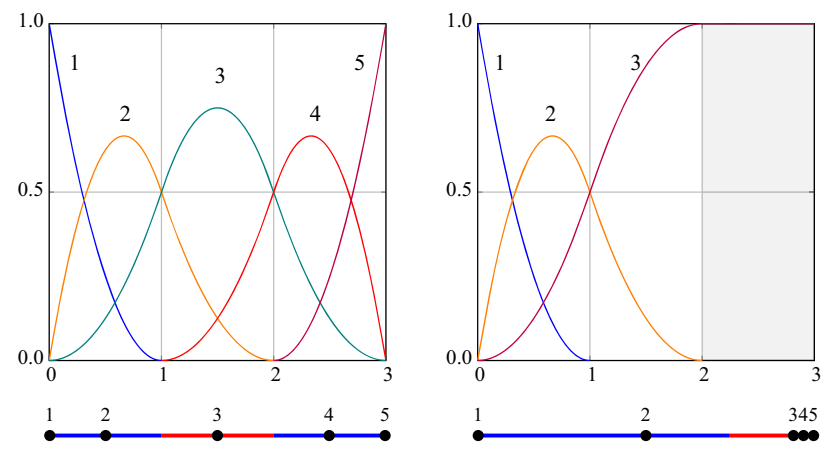

Fig. 2 Meshes and shape functions for the quadratic NURBS elements. The left mesh, which we see as the "parent" mesh, is for a wider stage of the gap. The right mesh is for when the gap is narrow and we reduce the number of control points to three by TC collapse. This results in two elements that are not desirable. The gray zone indicates the unused elements in the parametric space
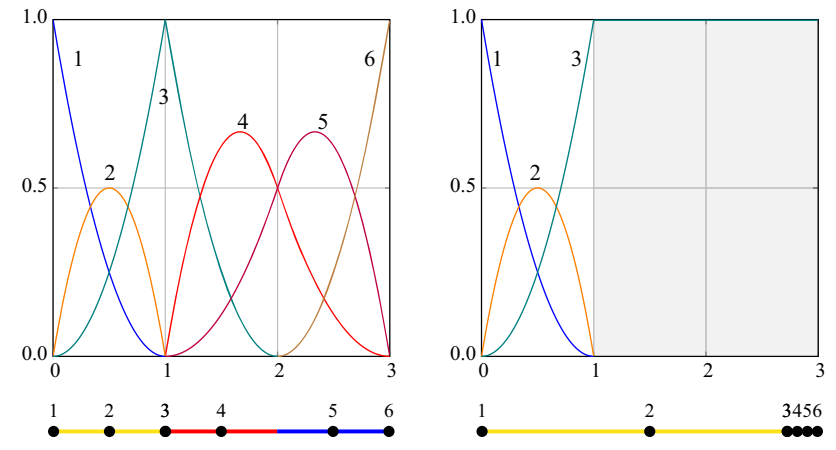

Fig. 3 Meshes and shape functions for the quadratic NURBS elements. The left mesh, which we see as the "parent" mesh, is for a wider stage of the gap. The right mesh is for when the gap is narrow and we reduce the number of control points to three by TC collapse. This results in one element with constant parametrization, which is what we wanted. The gray zone indicates the unused elements in the parametric space. We note that the parent mesh has a special element, which introduces a $C^{0}$-continuity

Remark 1 The quadratic-NURBS framework is not suitable for creating slaves to reduce the number of control points. A better framework would involve knot removal, which would basically require knot changes in time. However, that is beyond the scope of NURBS and may involve ST elements that are not tensor-products.

In the meantime, we propose a simpler solution, explained in Fig. 3. This solution includes accepting a $C^{0}$-continuity in the mesh.

\section{2D test computation with a straight channel}

We test the process of reducing the number of control points with the ST-SI-TC. We use an SI at one of the horizontal boundaries. The SI has an important role in a typical use of the ST-SI-TC. It enables contact location change and contact sliding. It also enables changing the location, with respect to the solid surface, where we reduce the number of control points.

\subsection{Problem setup}

Figure 4 shows the domain size and the boundary conditions. To test the SI part of the ST-SI-TC, we have an SI at the bottom boundary. The domain represents a narrow gap between two solid surfaces, after the SI, with TC collapse, coincides with the bottom solid surface. The flow is from left to right, and at the top and bottom boundaries we have no-slip condition. Because the bottom solid surface coincides with the SI, the no-slip condition is enforced weakly there. The Reynolds number is 1.0 . 


\subsection{Meshes}

Having a special quadratic NURBS element as explained in Fig. 3 can be accomplished in two ways. In the first way, shown in Fig. 5, we have one layer of special quadratic NURBS elements near the top solid surface. In the second way, shown in Fig. 6, the layer of special quadratic NURBS elements are just above the SI. In both cases, the parent mesh has elements below the SI, but the physical mesh will not. How the elements in these two cases collapse with the TC mechanism is explained in Figs. 7 and 8.

Remark 2 In theory, collapsing elements to the SI as shown in Fig. 7 is acceptable. However, then in the implementation of the SI formulation, such as numerical integration, we have to use elements that are not neighboring the SI in the parent mesh. That is not convenient for parallel implementation. Therefore we use the way described in Figs. 6 and 8.

20 $\lceil 1.0$

Fig. 4 Domain size and boundary conditions. Red, blue, black, and orange indicate the inflow, outflow, wall, and the SI. The domain represents a narrow gap between two solid surfaces, after the SI, with TC collapse, coincides with the bottom solid surface. (Color figure online)

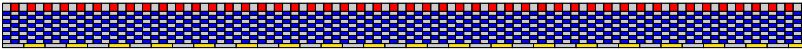

Fig. 5 Mesh-1. The yellow-gray elements, which are between the SI and the bottom solid surface, will collapse. The red-gray elements are the special quadratic NURBS elements we want to be left with, and they are placed at the top. The checkerboard coloring is for differentiating between the NURBS elements. (Color figure online)

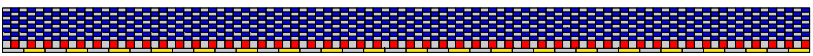

Fig. 6 Mesh-2. The yellow-gray elements, which are between the SI and the bottom solid surface, will collapse. The red-gray elements are the special quadratic NURBS elements we want to be left with, and they are placed just above the SI. The checkerboard coloring is for differentiating between the NURBS elements. (Color figure online)

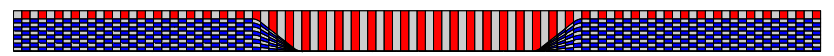

Fig. 7 Mesh-1-reduced. At the center part, the blue-gray elements collapse to the SI, and only the special quadratic NURBS elements remain, expanding to the SI. The checkerboard coloring is for differentiating between the NURBS elements. (Color figure online)

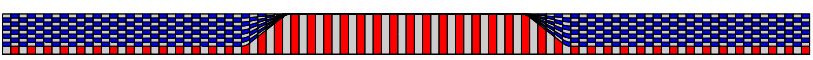

Fig. 8 Mesh-2-reduced. At the center part, the blue-gray elements collapse to the top solid surface, and only the special quadratic NURBS elements remain, expanding to the top solid surface. The checkerboard coloring is for differentiating between the NURBS elements. (Color figure online)
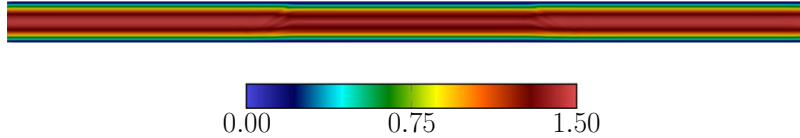

Fig. 9 Mesh-2-reduced. Velocity magnitude

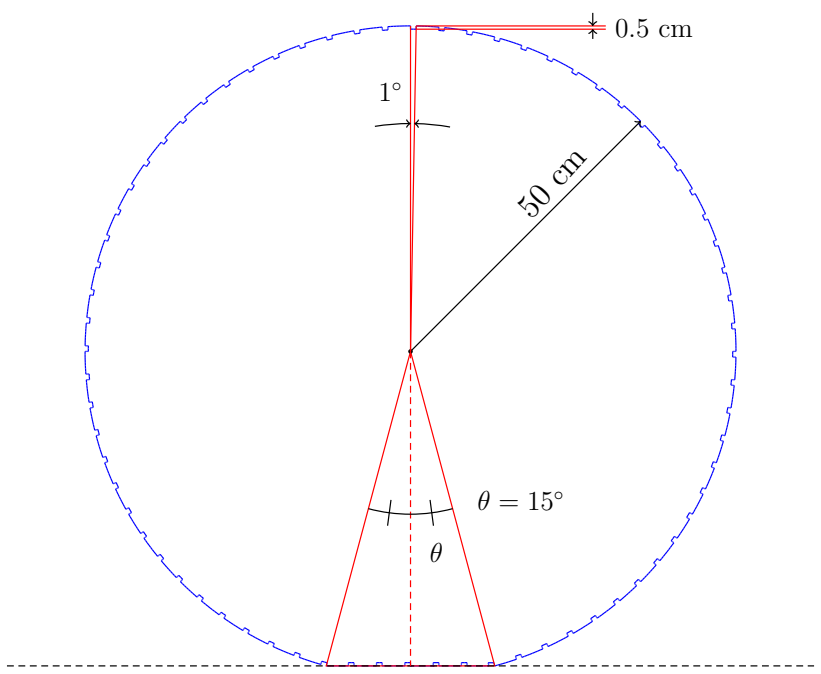

Fig. 10 A 2D tire model with grooves. There is a groove for every $5^{\circ}$. The deformation region is the circular sector with central angle $30^{\circ}$

\subsection{Computation}

We use Mesh-2-reduced. The method is the ST-VMS, and the stabilization parameters are those given by Eqs. (4)-(9) in [1] . The number of nonlinear iterations per time step is 3 , and the number of GMRES [144] iterations per nonlinear iteration is 100 . Figure 9 shows the velocity magnitude. The mesh transitions are handled well, resulting in a good solution.

\section{2D test computations with different contact representations}

We test our method on a multiscale problem in $2 \mathrm{D}$, with different ways of handling the region near the contact.

\subsection{Problem setup}

A 2D tire model with a groove for every $5^{\circ}$ is in contact with a planar solid surface and undergoes deformation (see Fig. 10). The contact angle $2 \theta=30^{\circ}$. The rotation speed corresponds to a linear speed of $U=100 \mathrm{~km} / \mathrm{h}$ at the undeformed tire periphery. There is no slip between the spinning and planar surfaces. The speed of the planar surface becomes

$U_{0}=\frac{\sin \theta}{\theta} U$, 


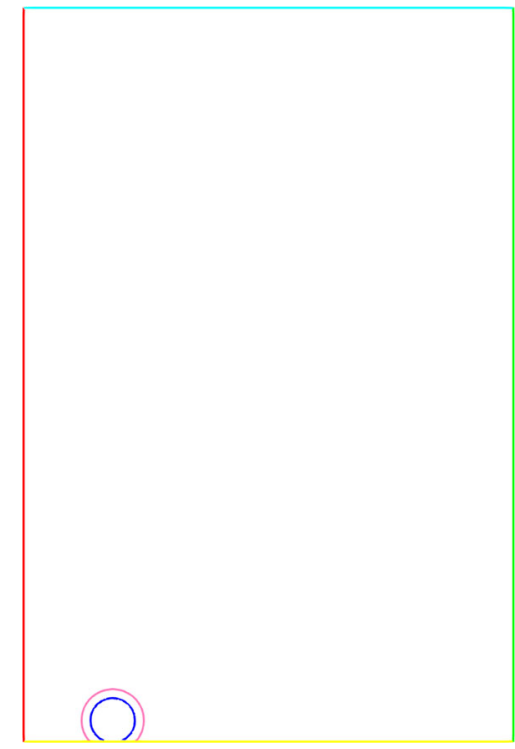

Fig. 11 A 2D tire model with grooves. Computational domain and boundary conditions. The left (red) and bottom (yellow) boundaries are the inflow and the moving planar surface, where the velocity is $U_{0}$. The innermost (blue) circle is the spinning surface, where the velocity is $U$. The larger (pink) circle is the SI. The bottom of the SI is coinciding with the planar surface and the interface of the spinning and planar surfaces. The conditions at the right (green) and upper (cyan) boundaries are stress-free and slip, respectively. (Color figure online)

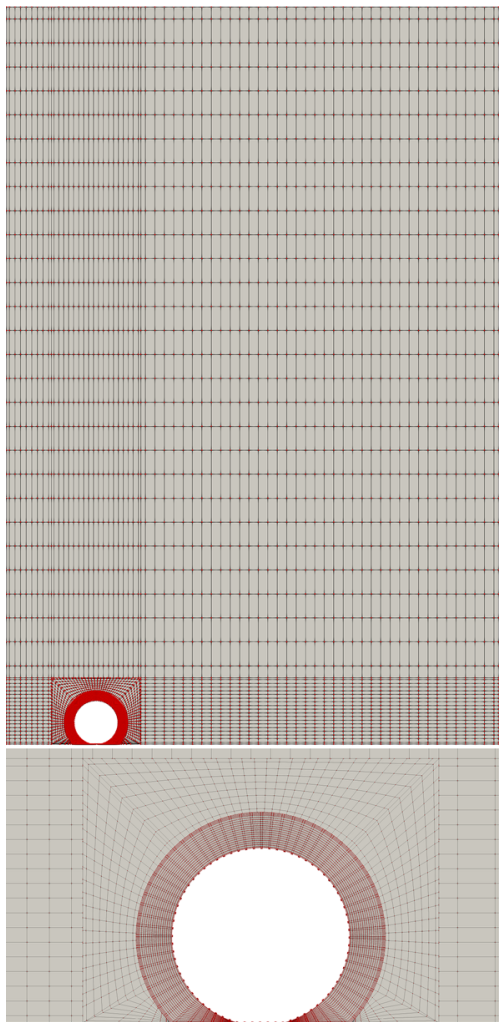

Fig. 12 A 2D tire model with grooves. Control mesh. Full view and focus view around the tire

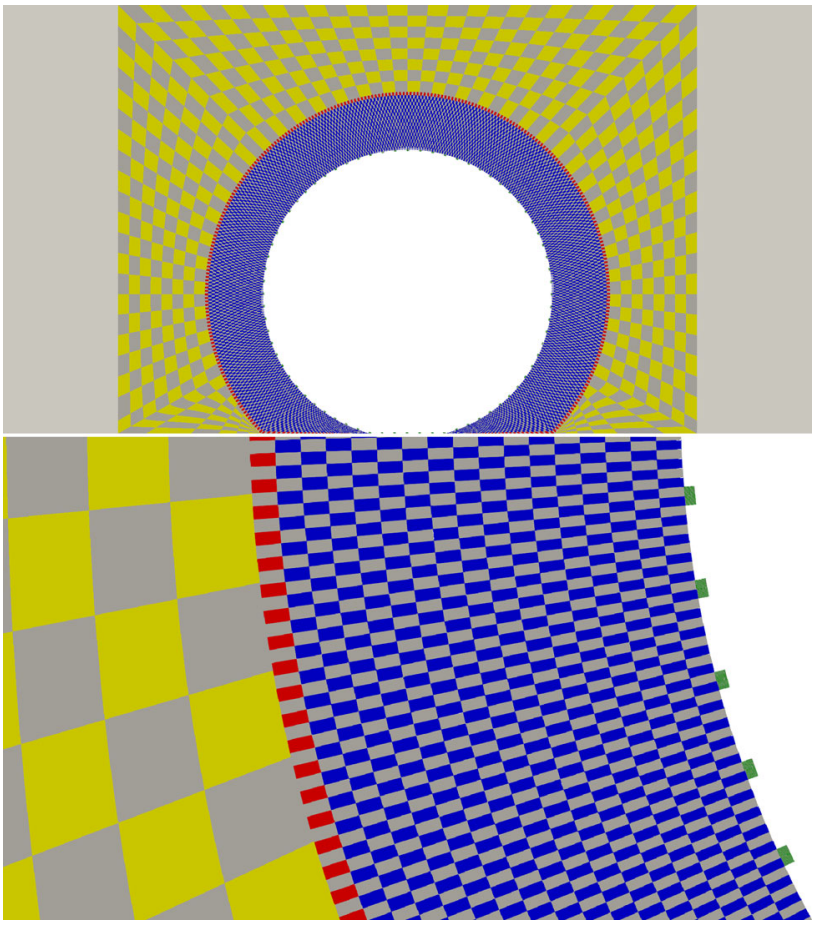

Fig. 13 A 2D tire model with grooves. The domain and elements near the tire. The gray and yellow-gray areas are the stationary domains, and the area with the other colors is the rotating domain. The red-gray elements are the special quadratic NURBS elements. Green-gray indicate the groove elements. Blue-gray indicate the remaining elements. The checkerboard coloring is for differentiating between the NURBS elements. (Color figure online)

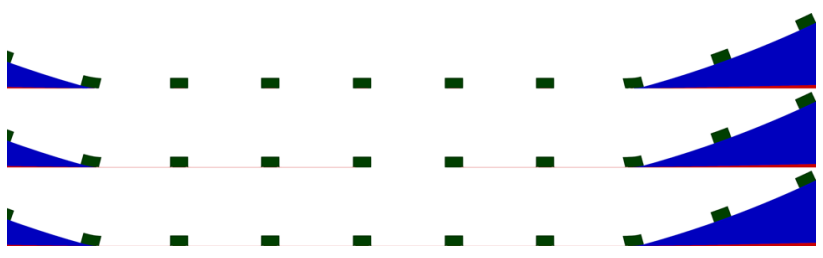

Fig. 14 A 2D tire model with grooves. Contact representations: "closing deisolation gaps (CDG)" (top), "untrapping the fluid with deisolation gaps (UFDG)" (middle), and "all deisolation gaps (ADG)" (bottom)

giving $U_{0}=98.86 \mathrm{~km} / \mathrm{h}$. The density and kinematic viscosity of the air are $1.205 \mathrm{~kg} / \mathrm{m}^{3}$ and $1.822 \times 10^{-5} \mathrm{~m}^{2} / \mathrm{s}$.

\subsection{Computational domain, boundary conditions and mesh}

Figure 11 shows the computational domain and boundary conditions. The domain size is $11.00 \times 16.48 \mathrm{~m}^{2}$. The left boundary is $2.0 \mathrm{~m}$ from the tire center. We have no-slip condition on the planar and tire surfaces. The no-slip condition is enforced weakly on the part of the planar surface coinciding with the SI. We use quadratic NURBS. Figure 12 shows the 


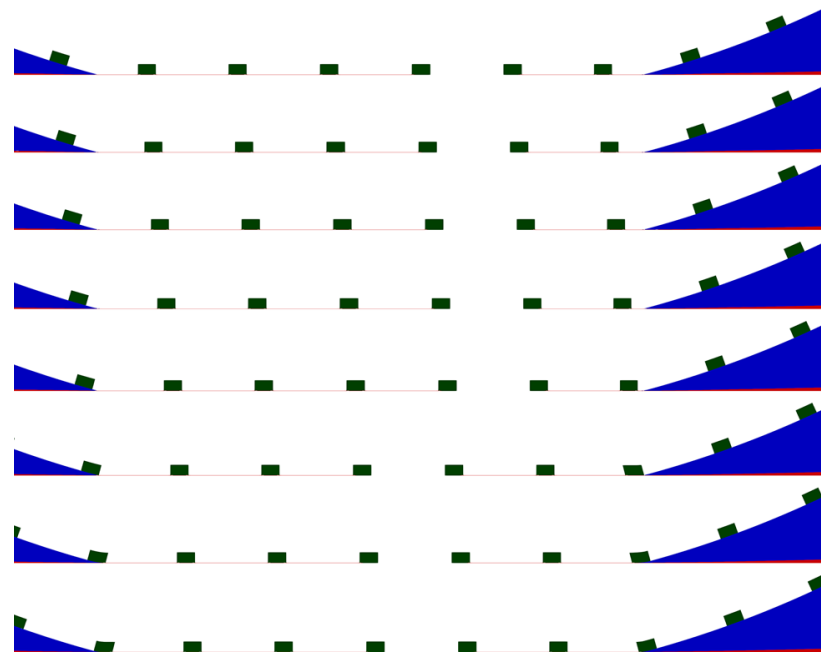

Fig. 15 A 2D tire model with grooves. Mesh motion for UFDG. We see that the full-contact zone switches (see the 5th and 6th frames)
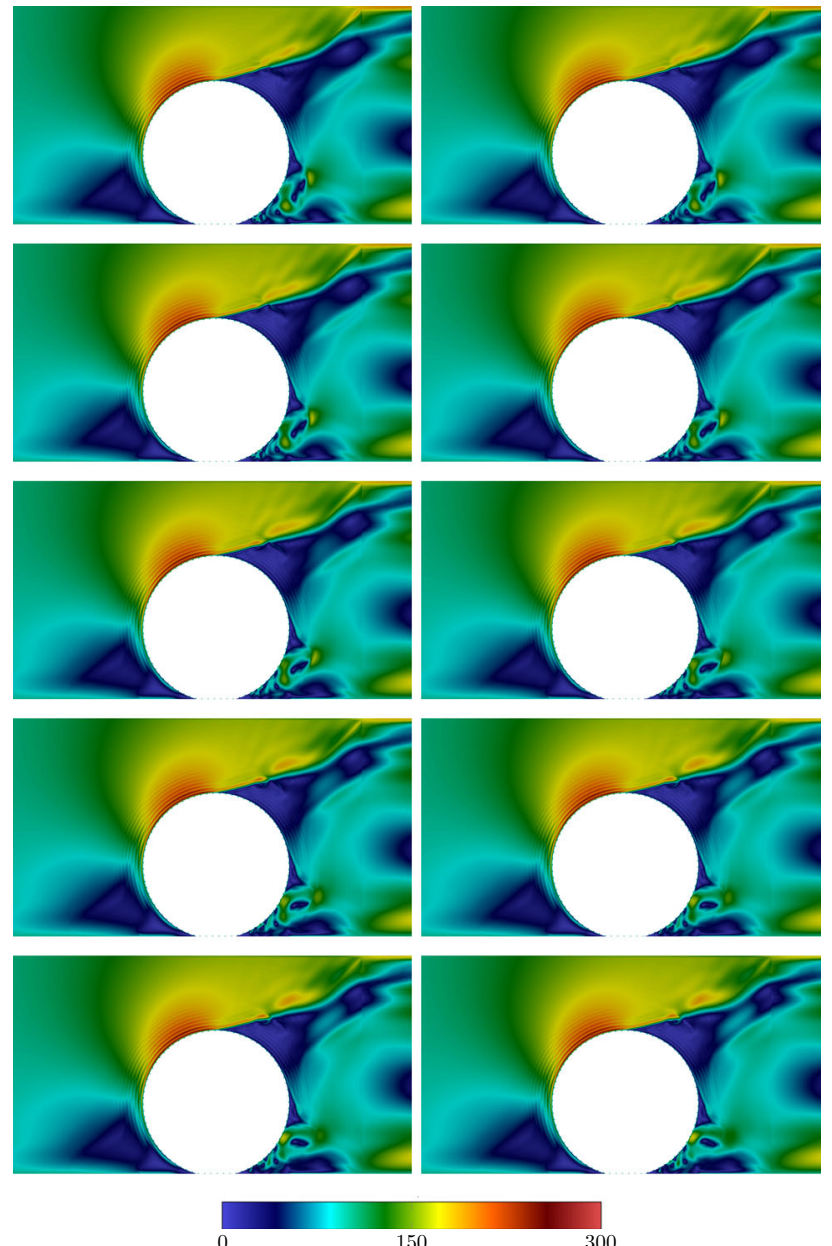

Fig. 16 A 2D tire model with grooves. Velocity magnitude $(\mathrm{km} / \mathrm{h})$ for $\mathrm{CDG}$ near the contact region
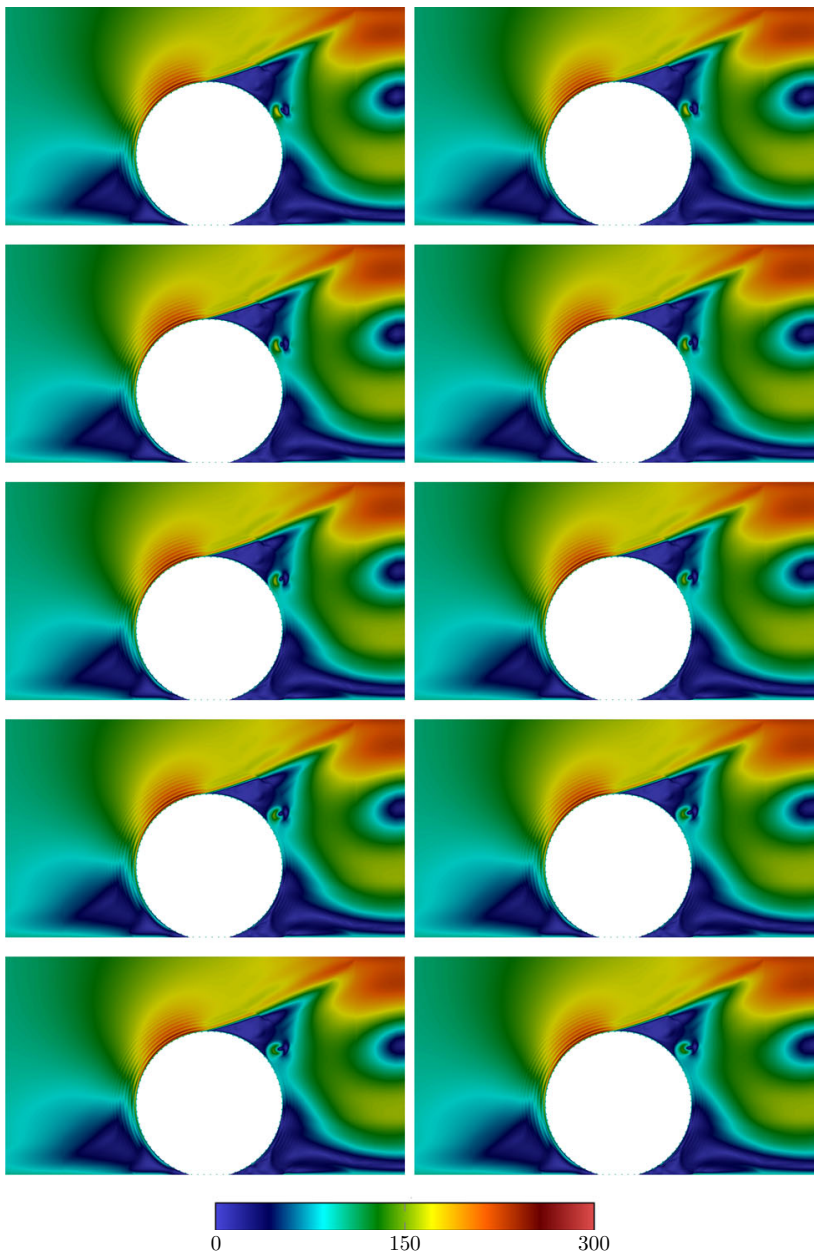

Fig. 17 A 2D tire model with grooves. Velocity magnitude $(\mathrm{km} / \mathrm{h})$ for UFDG near the contact region

control mesh. The number of control points is 26,902 , and the number of elements is 18,348 .

Figure 13 shows the domain and elements near the tire. The number of elements in the circumferential direction is 720. In the rotating domain, we have three regions: the region with special quadratic NURBS elements, the grooves, which have 2 elements in the circumferential direction and 18 elements in the radial direction, and the rest of the domain, which has 16 elements in the radial direction.

We consider three types of the contact representations: "closed deisolation gaps (CDG)," "untrapping the fluid with deisolation gaps (UFDG)," and "all deisolation gaps (ADG)." Figure 14 shows each representation.

The CDG case has no gaps, and the fluid is trapped in the grooves. The UFDG case has only one full-contact zone. The rest of the gap has a height of $0.1 \mathrm{~mm}$. The ADG case does not have a full-contact zone at all and the gap height is the same as in the UFDG case. Figure 15 shows the mesh motion for UFDG. 

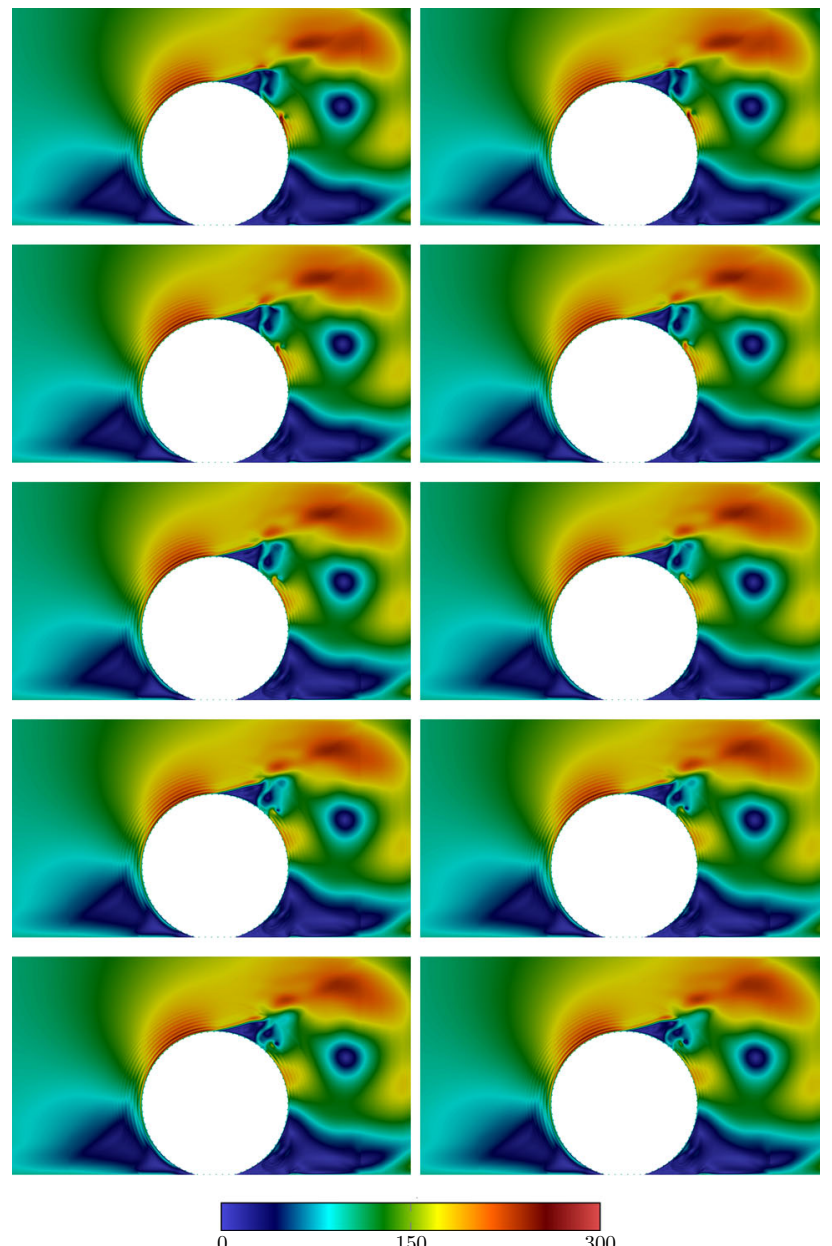

Fig. 18 A 2D tire model with grooves. Velocity magnitude $(\mathrm{km} / \mathrm{h})$ for ADG near the contact region

\subsection{Computational conditions}

In all three computations, the method is the ST-SUPS, and the stabilization parameters are those given by Eqs. (4)-(9) in [1]. There are 1000 time steps per rotation, which is equivalent to a time-step size of $1.131 \times 10^{-4} \mathrm{~s}$. The number of nonlinear iterations per time step is 3 , and the number of GMRES iterations per nonlinear iteration is 300 .

\subsection{Results and discussion}

Figures 16, 17, and 18 show the ninth rotation. The common point for all three cases is that the flow is affected by the fluid in the groove. In front of the tire the flow fields are comparable in all three cases. At the back of the tire the flow fields are close between UFDG and ADG, but CDG is quite different. This indicates that the last contact zone at the back of the tire makes a big difference.
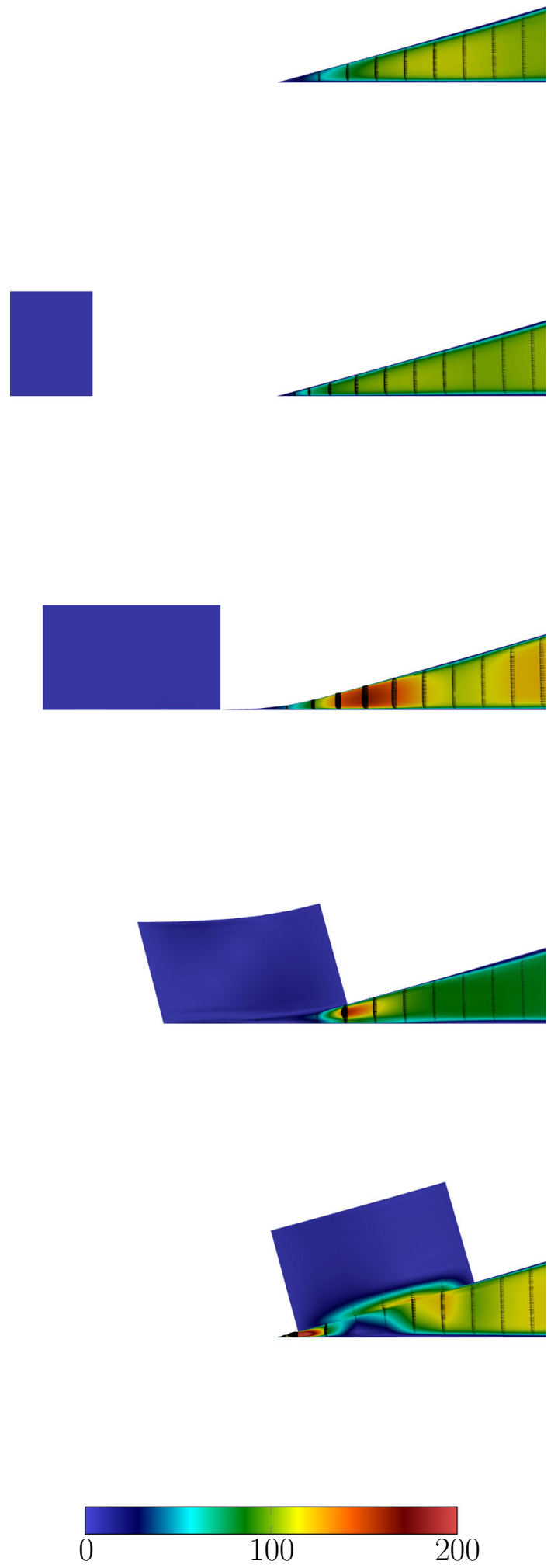

Fig. 19 A 2D tire model with grooves. Relative-to-ground velocity magnitude $(\mathrm{km} / \mathrm{h})$ for $\mathrm{CDG}$ near the contact region. The black vectors indicate the velocity 

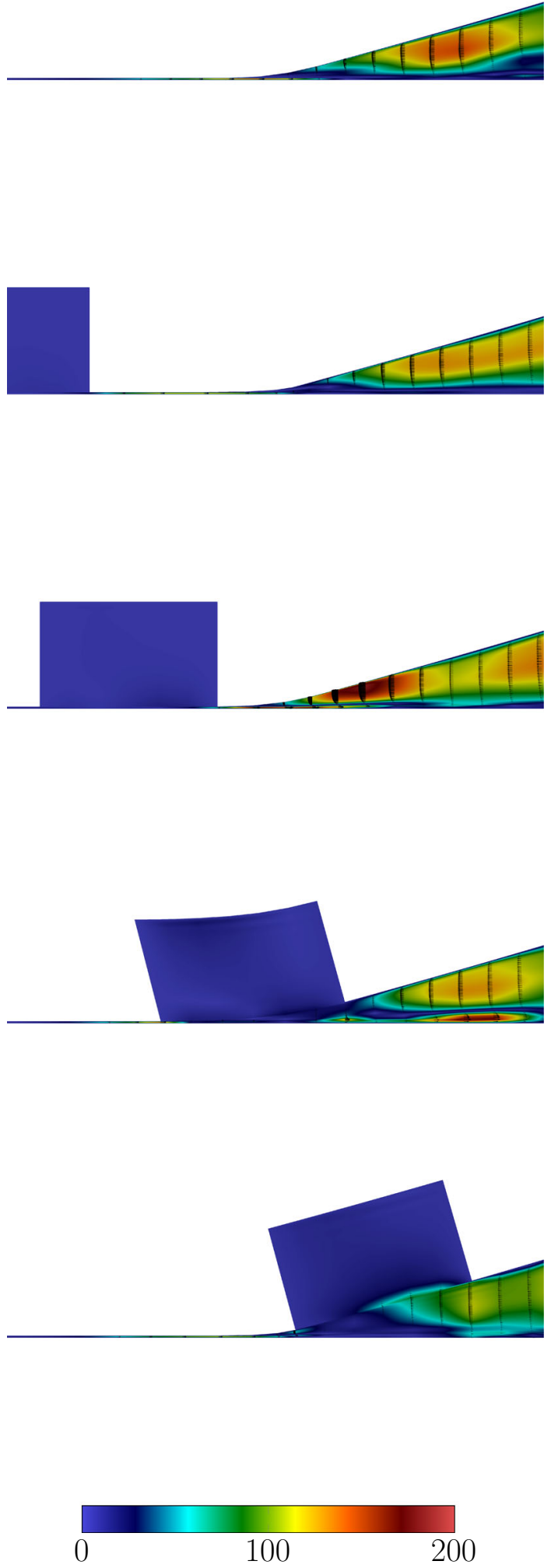

Fig. 20 A 2D tire model with grooves. Relative-to-ground velocity magnitude $(\mathrm{km} / \mathrm{h})$ for UFDG near the contact region. The black vectors indicate the velocity
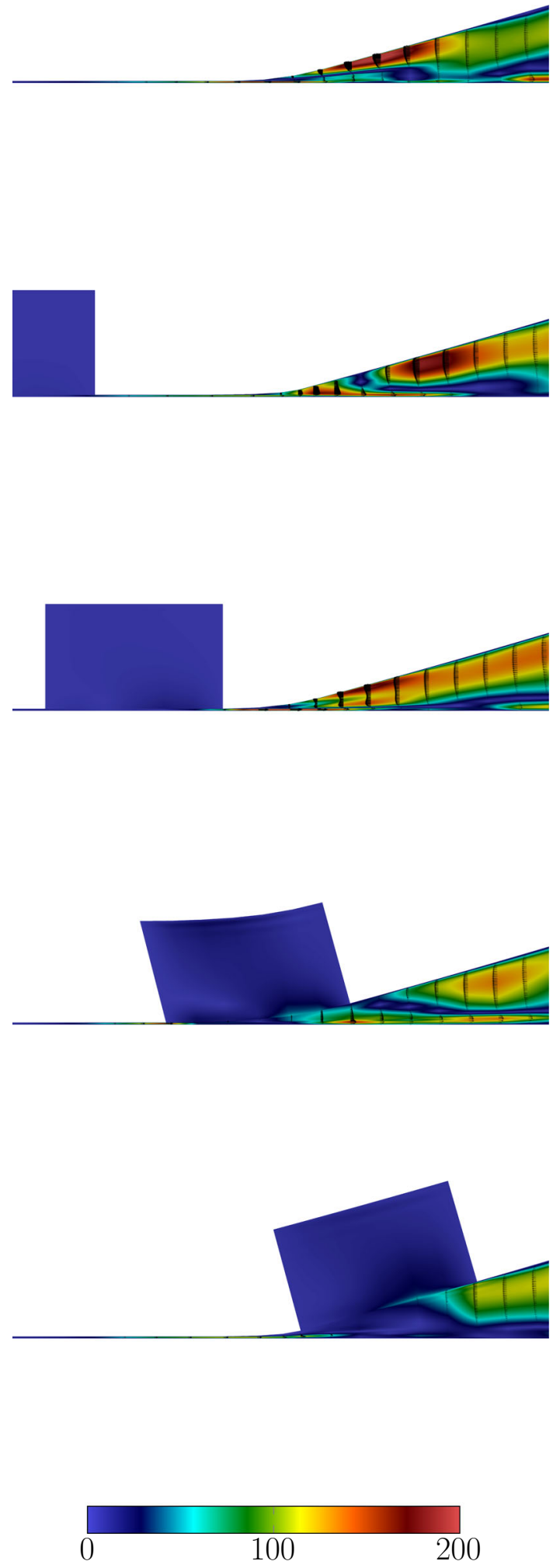

Fig. 21 A 2D tire model with grooves. Relative-to-ground velocity magnitude $(\mathrm{km} / \mathrm{h})$ for ADG near the contact region. The black vectors indicate the velocity 

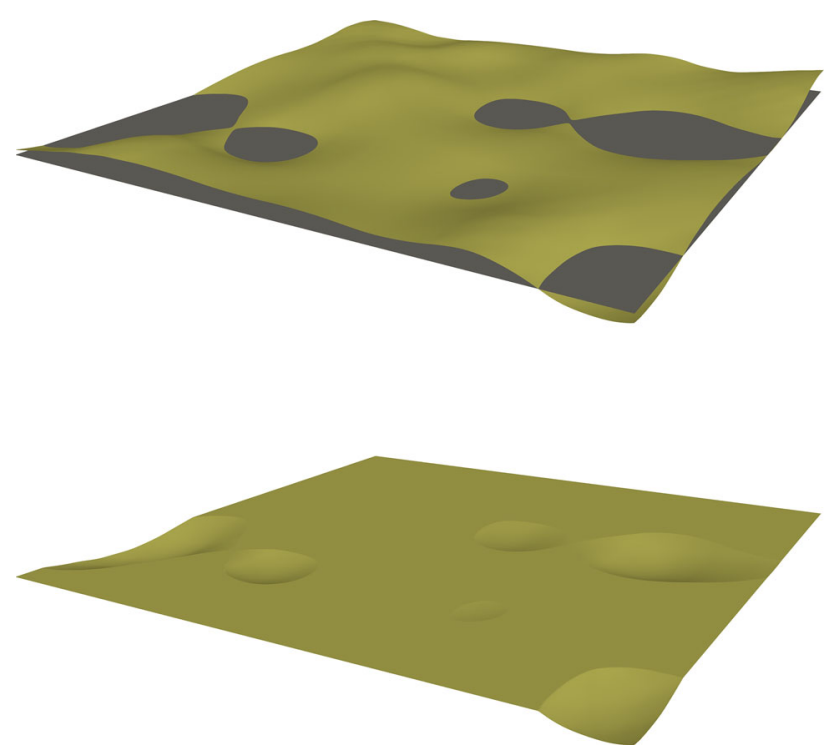

Fig. 22 Road surface with dents and bumps (top). The gray plane represents the tire surface. Road surface with dents only (bottom)

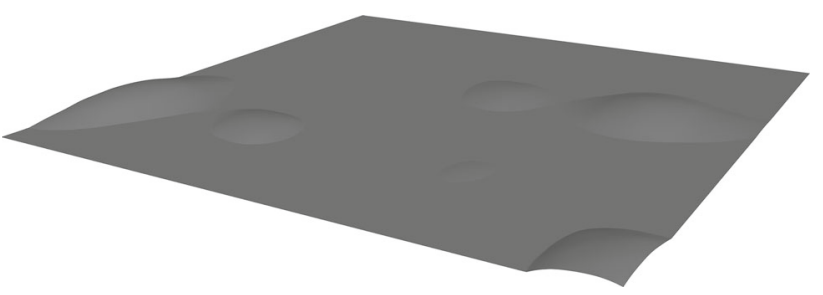

Fig. 23 Modified tire surface representing the road dents
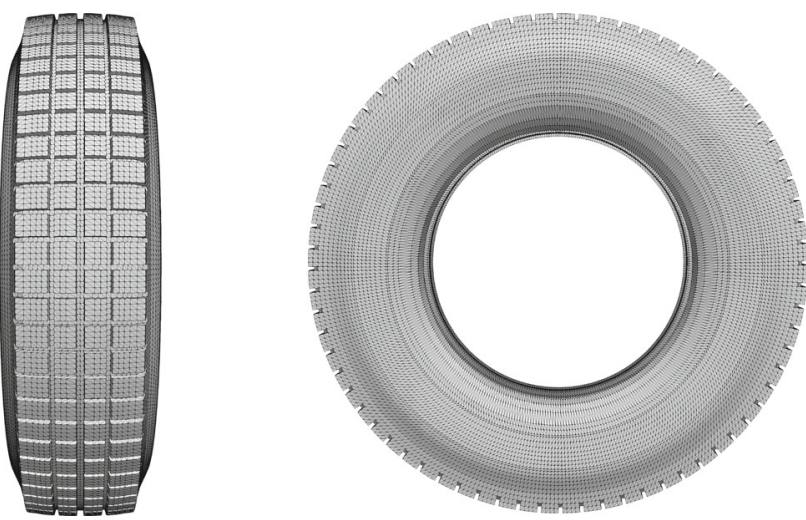

Fig. 24 Tire aerodynamics with actual tire geometry and road roughness. Tire model

We zoom in on the back of the contact region. Figures 19, 20 , and 21 show the velocity relative to the ground. In the CDG case, most of the flow is from right to left, as expected (tire is moving to the left). In the other two cases, however, some of the flow is from left to right. This is because the lower pressure at the back of the tire causes flow from the deisolation gap.
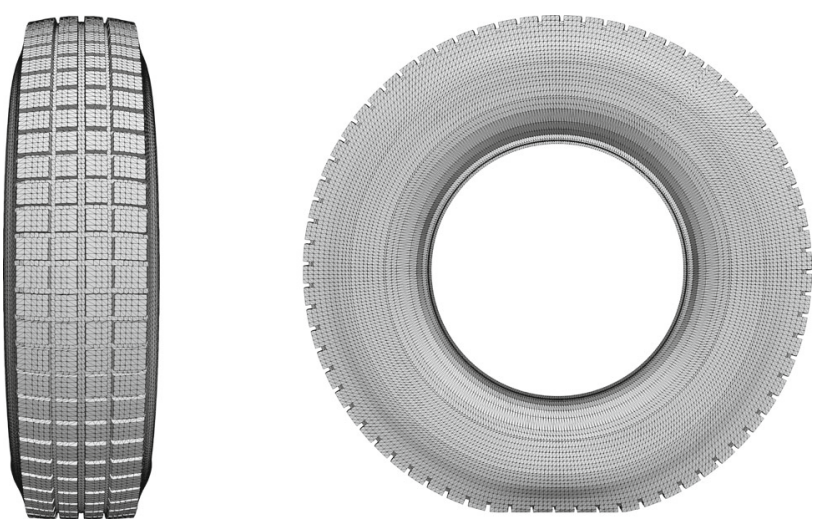

Fig. 25 Tire aerodynamics with actual tire geometry and road roughness. Deformed shape

Because this is a $2 \mathrm{D}$ model, the effect of the deisolation might be emphasized. We conclude that the ST-SI-TC can handle the contact and near contact representations very well.

\section{Tire aerodynamics with actual tire geometry and road roughness}

In this section, we present a tire-aerodynamics computation with actual tire geometry, road contact, tire deformation, road roughness and fluid film. Here we see the effect of the road roughness, with the dents decreasing the full-contact area, and that gives us a more realistic contact model.

\subsection{Representation of the road roughness}

We describe how we deal with the road roughness. Figure 22 shows the road surface and the plane representing the tire surface. Surface regions below the plane are dents and they contain air. In our mesh, the road representation is relatively coarse, not suitable for roughness modeling. Therefore, we flip the situation and the tire surface represents the dents, as shown in Fig. 23. In addition, we create paths on the tire surface to deisolate the dents.

Remark 3 We note that we do not model the dents coinciding with the grooves. That is because we assume that the groove depth is significantly larger than the dent depth.

\subsection{Tire model}

The tire model is shown in Fig. 24. The model is the same as the one in [1]. The diameter and width are $1.03 \mathrm{~m}$ and $260 \mathrm{~mm}$. There are three longitudinal grooves, and a transverse groove for every $5^{\circ}$. The depth and width of the grooves are $11.071 \mathrm{~mm}$ and $11.692 \mathrm{~mm}$ for the center groove, $10.974 \mathrm{~mm}$ and $7.177 \mathrm{~mm}$ for the side grooves, and 

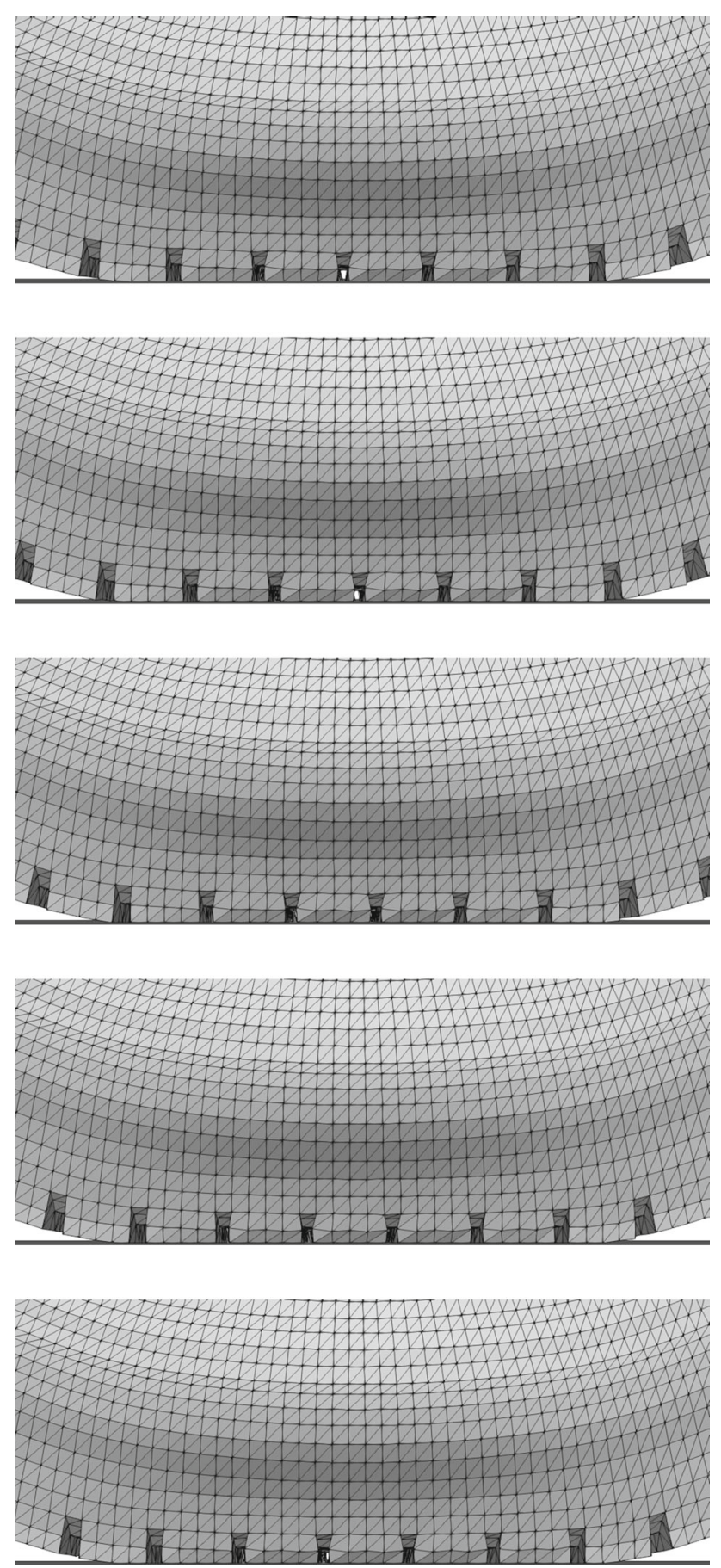

Fig. 26 Tire aerodynamics with actual tire geometry and road roughness. Tire deformation near the contact region at five instants of a $5^{\circ}$ rotation, provided by the tire company

$11.085 \mathrm{~mm}$ and $8.489 \mathrm{~mm}$ for the transverse grooves. Tire with the prescribed deformation is shown in Fig. 25.

The tire deformation is represented in time based on the deformation at five instants of a $5^{\circ}$ rotation, which was provided by the tire company. Figure 26 shows the tire deformation at those five instants. The deformation representation

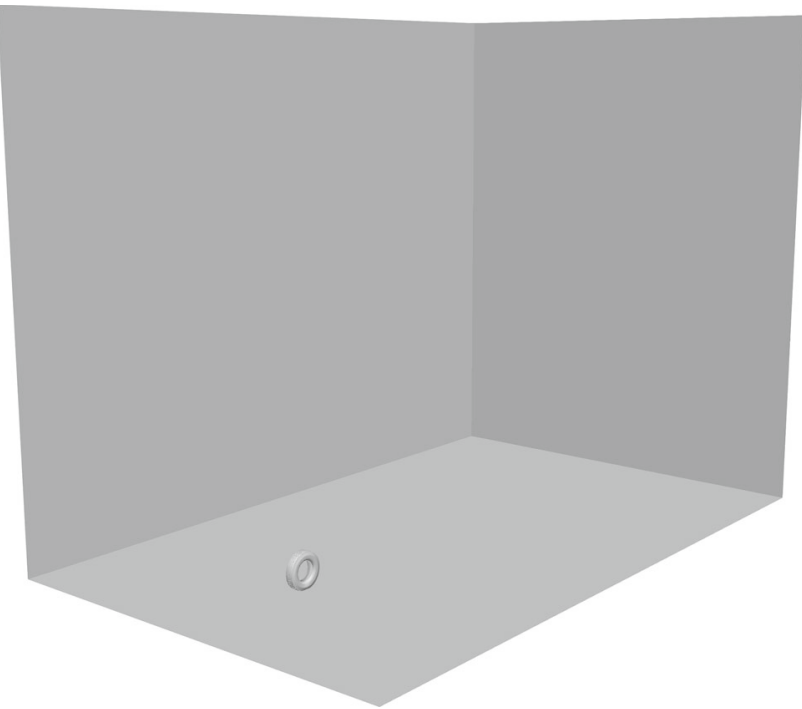

Fig. 27 Tire aerodynamics with actual tire geometry and road roughness. Computational domain

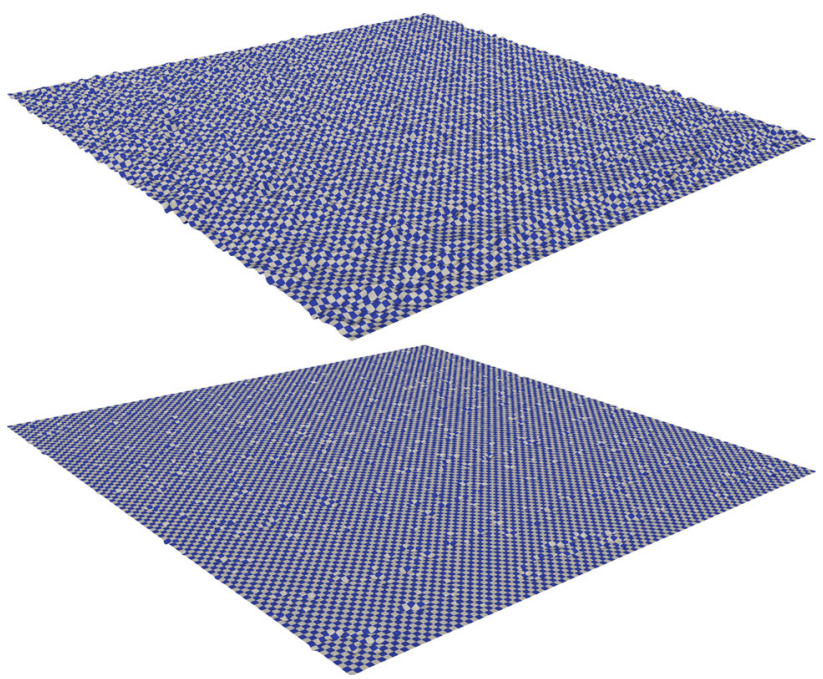

Fig. 28 Road surface (top) and road surface with dents only (bottom). For visualization purposes the roughness is amplified in the height direction

in time is with cubic NURBS basis functions and obtained by projection from the five-instant data. The projection is done with the ST-C [138]. The rotation speed corresponds to a linear speed of $100 \mathrm{~km} / \mathrm{h}$ at the undeformed tire periphery, and $U_{0}=98.86 \mathrm{~km} / \mathrm{h}$. The density and kinematic viscosity of the air are $1.205 \mathrm{~kg} / \mathrm{m}^{3}$ and $1.822 \times 10^{-5} \mathrm{~m}^{2} / \mathrm{s}$.

\subsection{Computational domain and boundary conditions}

The computational domain is shown in Fig. 27. The domain size is $12.000 \mathrm{~m}$ and $14.489 \mathrm{~m}$ in width and height, and $19.000 \mathrm{~m}$ in the flow direction. The tire is placed at $4.000 \mathrm{~m}$ 

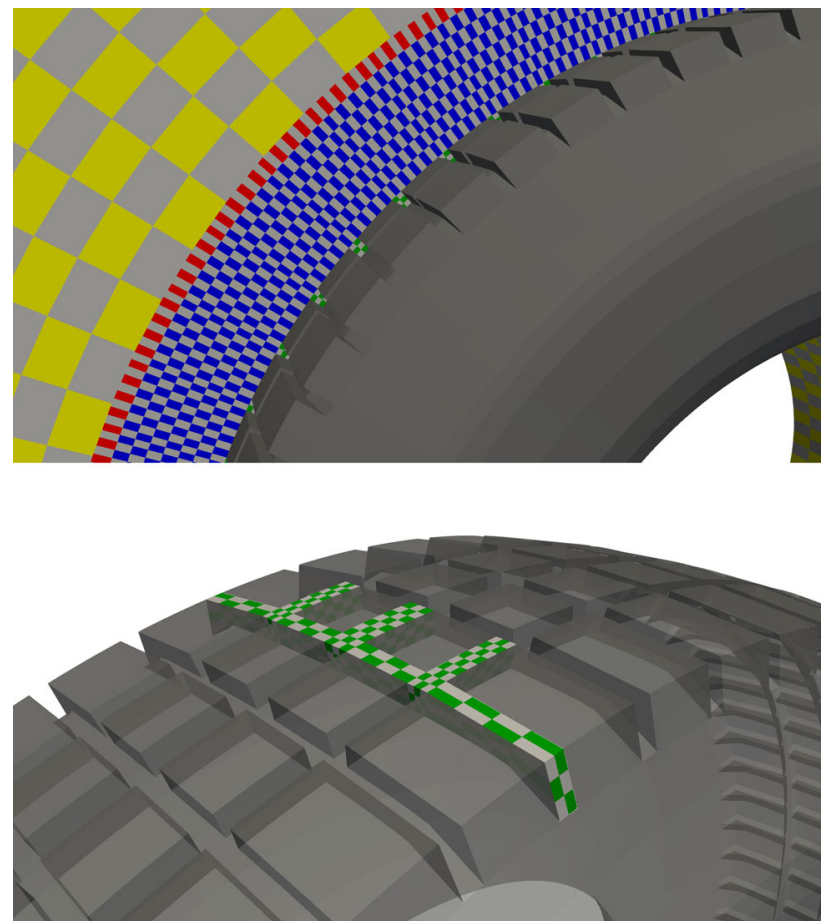

Fig. 29 Tire aerodynamics with actual tire geometry and road roughness. The mesh near the tire (top) and in the grooves (bottom). The red-gray elements are the special quadratic NURBS elements. Greengray indicate the groove elements. Blue-gray indicate the remaining elements. The checkerboard coloring is for differentiating between the NURBS elements. (Color figure online)

from the inflow boundary. The boundary conditions are 3D extensions of the conditions in the 2D model, with slip conditions on the boundary planes perpendicular to the tire axis. While we have no-slip condition on the road and tire surfaces, the condition is enforced weakly on the part of the road surface coinciding with the SI.

\subsection{Road roughness}

The road roughness is added in a random fashion, with $R_{\mathrm{a}}=0.21 \mathrm{~mm}$ and $S_{\mathrm{m}}=17.68 \mathrm{~mm}$, where $R_{\mathrm{a}}$ and $S_{\mathrm{m}}$ are $L_{1}$ norm of the surface position relative to the average surface position and mean spacing between the peaks. Figure 28 shows the road roughness. The dents are then projected to the tire surface. In addition, when a dent is isolated, we create a gap with a depth of $0.1 \mathrm{~mm}$ for the control point, serving as a path that deisolates the dent.

\subsection{Mesh and computational conditions}

The mesh, shown in Fig. 29, consists of 706,872 control points and 342,296 elements. The method is the ST-SUPS, and the stabilization parameters are those given by Eqs. (4)(9) in [1]. There are 1440 time steps per rotation, which is

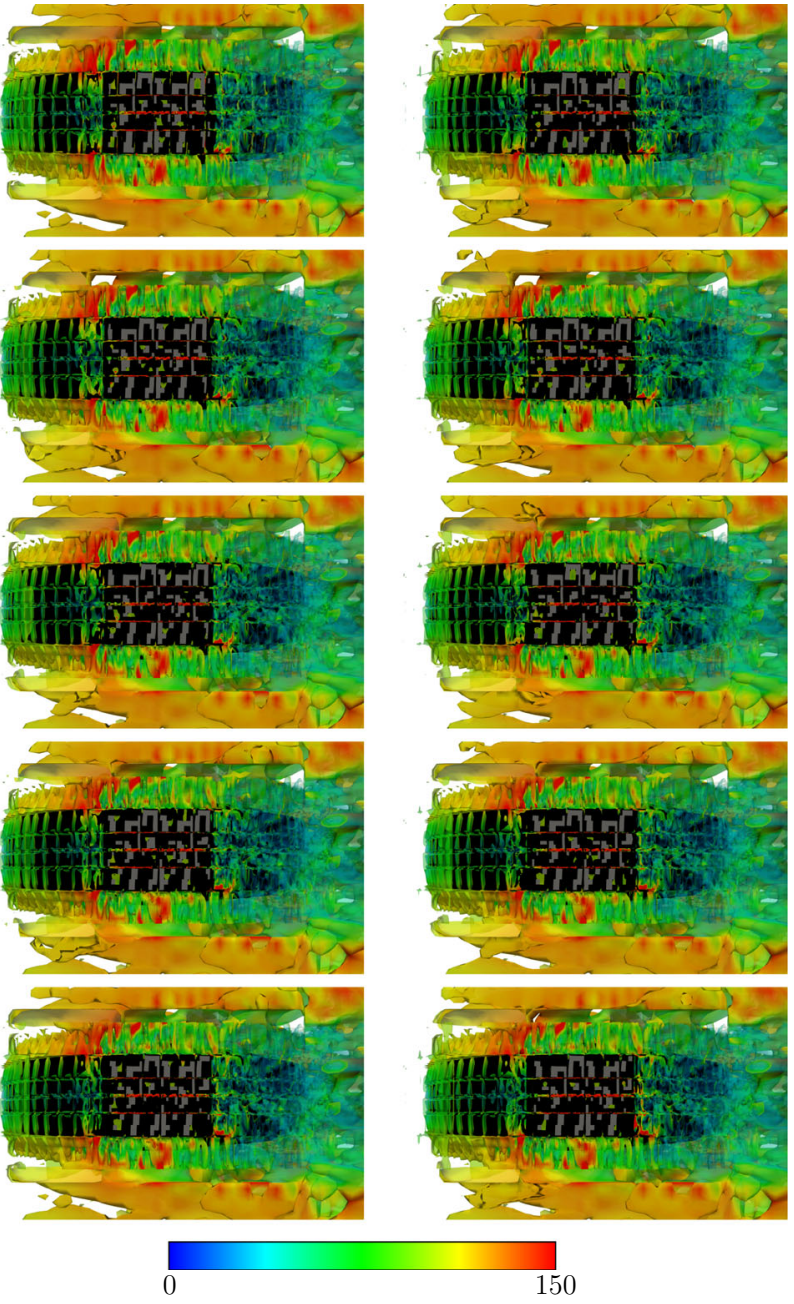

Fig. 30 Tire aerodynamics with actual tire geometry and road roughness. Isosurfaces corresponding to a positive value of the second invariant of the velocity gradient tensor, colored by the velocity magnitude $(\mathrm{km} / \mathrm{h})$, viewed from the bottom. The gray zones are the full-contact areas. (Color figure online)

equivalent to a time-step size of $8.09 \times 10^{-5} \mathrm{~s}$. The number of nonlinear iterations per time step is 3 , and the number of GMRES iterations per nonlinear iteration is 300 .

\subsection{Results}

Figure 30 shows the isosurfaces corresponding to a positive value of the second invariant of the velocity gradient tensor, colored by the velocity magnitude. Figure 31 shows positional-averaged shear stress acting on the tire surface, which is the fluid friction. As can be seen from the vectors included in the figure, there is flow in the deisolation gaps, to and from the dents, depending on the tire deformation pattern. 

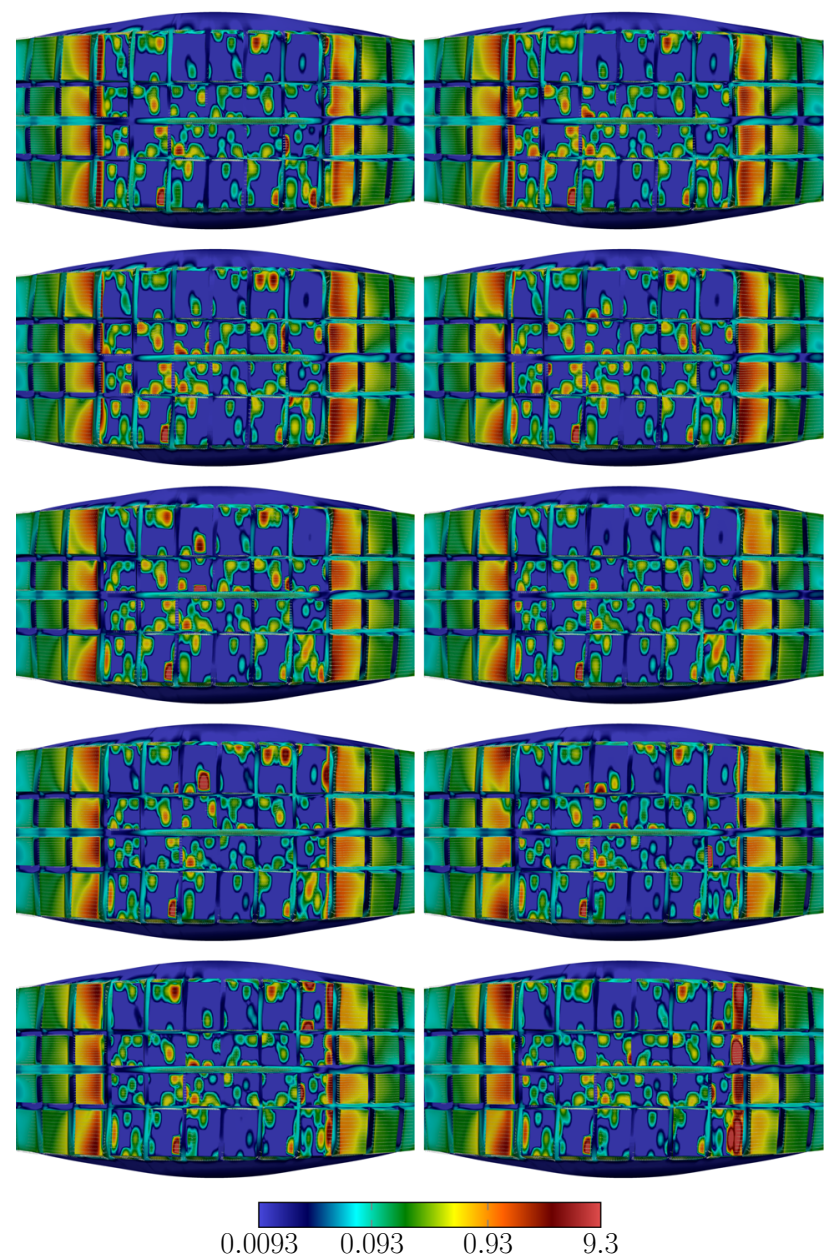

0.93

9.3

Fig. 31 Tire aerodynamics with actual tire geometry and road roughness. Positional-averaged shear stress $(\mathrm{Pa})$ acting on the tire surface. The vectors included indicate only the direction of the shear stress

\section{Concluding remarks}

Extending the work that addressed the challenges associated with computational analysis of tire aerodynamics with actual tire geometry, road contact and tire deformation, in this article we included and addressed the computational challenges associated with the road roughness and the fluid film between the tire and the road. The challenges include (i) the complexity of an actual tire geometry with longitudinal and transverse grooves, (ii) the spin of the tire, (iii) maintaining accurate representation of the boundary layers near the tire while being able to deal with the flow-domain topology change created by the road contact, (iv) the turbulent nature of the flow, (v) dealing with the fluid trapped between the tire and road surfaces, (vi) maintaining the cost effectiveness and generality of the computational method as we bring solution to fluid films embedded in the flow domain, and (vii) representing the road roughness.
The ST computational method ST-SI-TC-IGA does most of the heavy lifting in overcoming the computational challenges. The core component of the ST-SI-TC-IGA is the ST-VMS, and the other key components are the ST-SI, ST-TC and ST-IGA. The challenge created by the turbulent nature of the flow is addressed with the VMS feature of the ST-VMS. The moving-mesh feature of the ST framework enables highresolution flow computation near the air-tire interfaces as the tire rotates. These two features are enhanced with the higher-order accuracy of the ST framework. With the STSI, we are able to do moving-mesh computations with the tire spinning. The mesh covering the tire spins with it, and the SI between the spinning mesh and the rest of the mesh accurately connects the two sides of the solution. With the ST-TC, we are able to do moving-mesh computations even with the TC created by the contact between the tire and the road. This enables dealing with the contact while maintaining high-resolution flow representation near the tire. Integration of the ST-SI and ST-TC enables high-resolution flow representation even though parts of the SI are coinciding with the tire and road surfaces. It also enables dealing with the tire-road contact location change and contact sliding. Integration of the ST-IGA with the ST-SI and ST-TC not only enables a more accurate representation of the tire geometry and increased accuracy in the flow solution, but also keeps the element density in the tire grooves and in the narrow spaces near the contact areas at a reasonable level.

The additional methods include a method for creating deisolation paths for the trapped fluid, a method for reducing the number of control points as a space occupied by the fluid shrinks down to a narrow gap, and a method for representing the road roughness without increasing the mesh resolution on the road surface.

We first presented a 2D test computation for a straight channel. This test showed that mesh transitions between the zones with multiple elements and a single element across a gap are handled well. Then we presented, in the context of a simple 2D tire model, test computations with different contact representations. These tests demonstrated the significance of the deisolation paths. The last computation was with a 3D tire model with actual tire geometry and road roughness. This computation showed that the integrated set of ST methods are fully capable of dealing with essentially all the complexities of real-world tire aerodynamics. Overall, the computations presented show the effectiveness of the integrated set of ST methods targeting tire aerodynamics.

Acknowledgements This work was supported (first and second authors) in part by Grant-in-Aid for Challenging Exploratory Research 16K13779 from JSPS; Grant-in-Aid for Scientific Research (S) 26220002 from the Ministry of Education, Culture, Sports, Science and Technology of Japan (MEXT); and Rice-Waseda research agreement. This work was also supported (first author) in part by Grant-in-Aid for JSPS Research Fellow 17J10893. The computational method parts of the work were 
also supported (third author) in part by ARO Grant W911NF-17-10046 and Top Global University Project of Waseda University. The tire deformation used in Sect. 11 was provided by Bridgestone.

Open Access This article is distributed under the terms of the Creative Commons Attribution 4.0 International License (http://creativecomm ons.org/licenses/by/4.0/), which permits unrestricted use, distribution, and reproduction in any medium, provided you give appropriate credit to the original author(s) and the source, provide a link to the Creative Commons license, and indicate if changes were made.

\section{References}

1. Kuraishi T, Takizawa K, Tezduyar TE (2019) Tire aerodynamics with actual tire geometry, road contact and tire deformation. Comput Mech 63:1165-1185. https://doi.org/10.1007/s00466018-1642-1

2. Takizawa K, Tezduyar TE, Terahara T, Sasaki T (2017) Heart valve flow computation with the integrated space-time VMS, slip interface, topology change and isogeometric discretization methods. Comput Fluids 158:176-188. https://doi.org/10.1016/j. compfluid.2016.11.012

3. Takizawa K, Tezduyar TE (2011) Multiscale space-time fluidstructure interaction techniques. Comput Mech 48:247-267. https://doi.org/10.1007/s00466-011-0571-z

4. Takizawa K, Tezduyar TE (2012) Space-time fluidstructure interaction methods. Math Models Methods Appl Sci 22(supp02):1230001. https://doi.org/10.1142/ S0218202512300013

5. Takizawa K, Tezduyar TE, Kuraishi T (2015) Multiscale ST methods for thermo-fluid analysis of a ground vehicle and its tires. Math Models Methods Appl Sci 25:2227-2255. https://doi.org/ $10.1142 / \mathrm{S} 0218202515400072$

6. Takizawa K, Tezduyar TE, Mochizuki H, Hattori H, Mei S, Pan L, Montel K (2015) Space-time VMS method for flow computations with slip interfaces (ST-SI). Math Models Methods Appl Sci 25:2377-2406. https://doi.org/10.1142/S0218202515400126

7. Takizawa K, Tezduyar TE, Kuraishi T, Tabata S, Takagi H (2016) Computational thermo-fluid analysis of a disk brake. Comput Mech 57:965-977. https://doi.org/10.1007/s00466-016-1272-4

8. Takizawa K, Tezduyar TE, Buscher A, Asada S (2014) Spacetime interface-tracking with topology change (ST-TC). Comput Mech 54:955-971. https://doi.org/10.1007/s00466-013-0935-7

9. Takizawa K, Tezduyar TE, Buscher A, Asada S (2014) Spacetime fluid mechanics computation of heart valve models. Comput Mech 54:973-986. https://doi.org/10.1007/s00466-014-1046-9

10. Takizawa K, Henicke B, Puntel A, Spielman T, Tezduyar TE (2012) Space-time computational techniques for the aerodynamics of flapping wings. J Appl Mech 79:010903. https://doi.org/10. $1115 / 1.4005073$

11. Takizawa K, Tezduyar TE, Otoguro Y, Terahara T, Kuraishi T, Hattori H (2017) Turbocharger flow computations with the spacetime isogeometric analysis (ST-IGA). Comput Fluids 142:15-20. https://doi.org/10.1016/j.compfluid.2016.02.021

12. Kuraishi T, Takizawa K, Tezduyar TE (2019) Space-time Isogeometric flow analysis with built-in Reynolds-equation limit. Math Models Methods Appl Sci 29:871-904. https://doi.org/10.1142/ S0218202519410021

13. Tezduyar TE (1992) Stabilized finite element formulations for incompressible flow computations. Adv Appl Mech 28:1-44. https://doi.org/10.1016/S0065-2156(08)70153-4

14. Tezduyar TE (2003) Computation of moving boundaries and interfaces and stabilization parameters. Int J Numer Methods Fluids 43:555-575. https://doi.org/10.1002/fld.505
15. Tezduyar TE, Sathe S (2007) Modeling of fluid-structure interactions with the space-time finite elements: solution techniques. Int J Numer Methods Fluids 54:855-900. https://doi.org/10.1002/ fld. 1430

16. Brooks AN, Hughes TJR (1982) Streamline upwind/PetrovGalerkin formulations for convection dominated flows with particular emphasis on the incompressible Navier-Stokes equations. Comput Methods Appl Mech Eng 32:199-259

17. Hughes TJR (1995) Multiscale phenomena: Green's functions, the Dirichlet-to-Neumann formulation, subgrid scale models, bubbles, and the origins of stabilized methods. Comput Methods Appl Mech Eng 127:387-401

18. Hughes TJR, Oberai AA, Mazzei L (2001) Large eddy simulation of turbulent channel flows by the variational multiscale method. Phys Fluids 13:1784-1799

19. Bazilevs Y, Calo VM, Cottrell JA, Hughes TJR, Reali A, Scovazzi G (2007) Variational multiscale residual-based turbulence modeling for large eddy simulation of incompressible flows. Comput Methods Appl Mech Eng 197:173-201

20. Bazilevs Y, Akkerman I (2010) Large eddy simulation of turbulent Taylor-Couette flow using isogeometric analysis and the residualbased variational multiscale method. J Comput Phys 229:34023414

21. Bazilevs Y, Calo VM, Hughes TJR, Zhang Y (2008) Isogeometric fluid-structure interaction: theory, algorithms, and computations. Comput Mech 43:3-37

22. Takizawa K, Bazilevs Y, Tezduyar TE (2012) Space-time and ALE-VMS techniques for patient-specific cardiovascular fluidstructure interaction modeling. Arch Comput Methods Eng 19:171-225. https://doi.org/10.1007/s11831-012-9071-3

23. Bazilevs Y, Hsu M-C, Takizawa K, Tezduyar TE (2012) ALEVMS and ST-VMS methods for computer modeling of windturbine rotor aerodynamics and fluid-structure interaction. Math Models Methods Appl Sci 22(supp02):1230002. https://doi.org/ 10.1142/S0218202512300025

24. Bazilevs Y, Takizawa K, Tezduyar TE (2013) Computational Fluid-Structure Interaction: Methods and Applications. Wiley, London ISBN 978-0470978771

25. Bazilevs Y, Takizawa K, Tezduyar TE (2013) Challenges and directions in computational fluid-structure interaction. Math Models Methods Appl Sci 23:215-221. https://doi.org/10.1142/ S0218202513400010

26. Bazilevs Y, Takizawa K, Tezduyar TE (2015) New directions and challenging computations in fluid dynamics modeling with stabilized and multiscale methods. Math Models Methods Appl Sci 25:2217-2226. https://doi.org/10.1142/S0218202515020029

27. Bazilevs Y, Takizawa K, Tezduyar TE (2019) Computational analysis methods for complex unsteady flow problems. Math Models Methods Appl Sci 29:825-838. https://doi.org/10.1142/ S0218202519020020

28. Kalro V, Tezduyar TE (2000) A parallel 3D computational method for fluid-structure interactions in parachute systems. Comput Methods Appl Mech Eng 190:321-332. https://doi.org/10.1016/ S0045-7825(00)00204-8

29. Bazilevs Y, Hughes TJR (2007) Weak imposition of Dirichlet boundary conditions in fluid mechanics. Comput Fluids 36:1226

30. Bazilevs Y, Michler C, Calo VM, Hughes TJR (2010) Isogeometric variational multiscale modeling of wall-bounded turbulent flows with weakly enforced boundary conditions on unstretched meshes. Comput Methods Appl Mech Eng 199:780-790

31. Hsu M-C, Akkerman I, Bazilevs Y (2012) Wind turbine aerodynamics using ALE-VMS: validation and role of weakly enforced boundary conditions. Comput Mech 50:499-511 
32. Bazilevs Y, Hughes TJR (2008) NURBS-based isogeometric analysis for the computation of flows about rotating components. Comput Mech 43:143-150

33. Hsu M-C, Bazilevs Y (2012) Fluid-structure interaction modeling of wind turbines: simulating the full machine. Comput Mech 50:821-833

34. Moghadam ME, Bazilevs Y, Hsia T-Y, Vignon-Clementel IE, Marsden AL (2011) A comparison of outlet boundary treatments for prevention of backflow divergence with relevance to blood flow simulations. Comput Mech 48:277-291. https://doi.org/10. 1007/s00466-011-0599-0

35. Bazilevs Y, Hsu M-C, Akkerman I, Wright S, Takizawa K, Henicke B, Spielman T, Tezduyar TE (2011) 3D simulation of wind turbine rotors at full scale. Part I: geometry modeling and aerodynamics. Int J Numer Methods Fluids 65:207-235. https:// doi.org/10.1002/fld.2400

36. Bazilevs Y, Hsu M-C, Kiendl J, Wüchner R, Bletzinger K-U (2011) 3D simulation of wind turbine rotors at full scale. Part II: fluid-structure interaction modeling with composite blades. Int J Numer Methods Fluids 65:236-253

37. Hsu M-C, Akkerman I, Bazilevs Y (2011) High-performance computing of wind turbine aerodynamics using isogeometric analysis. Comput Fluids 49:93-100

38. Bazilevs Y, Hsu M-C, Scott MA (2012) Isogeometric fluidstructure interaction analysis with emphasis on non-matching discretizations, and with application to wind turbines. Comput Methods Appl Mech Eng 249-252:28-41

39. Hsu M-C, Akkerman I, Bazilevs Y (2014) Finite element simulation of wind turbine aerodynamics: validation study using NREL phase VI experiment. Wind Energy 17:461-481

40. Korobenko A, Hsu M-C, Akkerman I, Tippmann J, Bazilevs Y (2013) Structural mechanics modeling and FSI simulation of wind turbines. Math Models Methods Appl Sci 23:249-272

41. Bazilevs Y, Takizawa K, Tezduyar TE, Hsu M-C, Kostov N, McIntyre S (2014) Aerodynamic and FSI analysis of wind turbines with the ALE-VMS and ST-VMS methods. Arch Comput Methods Eng 21:359-398. https://doi.org/10.1007/s11831-014-9119-7

42. Bazilevs Y, Korobenko A, Deng X, Yan J (2015) Novel structural modeling and mesh moving techniques for advanced FSI simulation of wind turbines. Int J Numer Methods Eng 102:766-783. https://doi.org/10.1002/nme.4738

43. Korobenko A, Yan J, Gohari SMI, Sarkar S, Bazilevs Y (2017) FSI simulation of two back-to-back wind turbines in atmospheric boundary layer flow. Comput Fluids 158:167-175. https://doi.org/ 10.1016/j.compfluid.2017.05.010

44. Korobenko A, Bazilevs Y, Takizawa K, Tezduyar TE (2018) Recent advances in ALE-VMS and ST-VMS computational aerodynamic and FSI analysis of wind turbines. In: Tezduyar TE (ed) Frontiers in computational fluid-structure interaction and flow simulation: research from lead investigators under forty-2018, modeling and simulation in science, engineering and technology. Springer, Berlin, pp 253-336. https://doi.org/10.1007/9783-319-96469-0_7 ISBN 978-3-319-96468-3

45. Korobenko A, Bazilevs Y, Takizawa K, Tezduyar TE (2018) Computer modeling of wind turbines: 1. ALE-VMS and ST-VMS aerodynamic and FSI analysis. Arch Comput Methods Eng, published online, https://doi.org/10.1007/s11831-018-9292-1

46. Korobenko A, Hsu M-C, Akkerman I, Bazilevs Y (2013) Aerodynamic simulation of vertical-axis wind turbines. J Appl Mech 81:021011. https://doi.org/10.1115/1.4024415

47. Bazilevs Y, Korobenko A, Deng X, Yan J, Kinzel M, Dabiri JO (2014) FSI modeling of vertical-axis wind turbines. J Appl Mech 81:081006. https://doi.org/10.1115/1.4027466

48. Yan J, Korobenko A, Deng X, Bazilevs Y (2016) Computational free-surface fluid-structure interaction with application to floating offshore wind turbines. Comput Fluids 141:155-174. https://doi. org/10.1016/j.compfluid.2016.03.008

49. Bazilevs Y, Korobenko A, Yan J, Pal A, Gohari SMI, Sarkar S (2015) ALE-VMS formulation for stratified turbulent incompressible flows with applications. Math Models Methods Appl Sci 25:2349-2375. https://doi.org/10.1142/S0218202515400114

50. Bazilevs Y, Korobenko A, Deng X, Yan J (2016) FSI modeling for fatigue-damage prediction in full-scale wind-turbine blades. $\mathrm{J}$ Appl Mech 83(6):061010

51. Bazilevs Y, Calo VM, Zhang Y, Hughes TJR (2006) Isogeometric fluid-structure interaction analysis with applications to arterial blood flow. Comput Mech 38:310-322

52. Bazilevs Y, Gohean JR, Hughes TJR, Moser RD, Zhang Y (2009) Patient-specific isogeometric fluid-structure interaction analysis of thoracic aortic blood flow due to implantation of the Jarvik 2000 left ventricular assist device. Comput Methods Appl Mech Eng 198:3534-3550

53. Bazilevs Y, Hsu M-C, Benson D, Sankaran S, Marsden A (2009) Computational fluid-structure interaction: methods and application to a total cavopulmonary connection. Comput Mech 45:77-89

54. Bazilevs Y, Hsu M-C, Zhang Y, Wang W, Liang X, Kvamsdal T, Brekken R, Isaksen J (2010) A fully-coupled fluid-structure interaction simulation of cerebral aneurysms. Comput Mech 46:3-16

55. Bazilevs Y, Hsu M-C, Zhang Y, Wang W, Kvamsdal T, Hentschel S, Isaksen J (2010) Computational fluid-structure interaction: methods and application to cerebral aneurysms. Biomech Model Mechanobiol 9:481-498

56. Hsu M-C, Bazilevs Y (2011) Blood vessel tissue prestress modeling for vascular fluid-structure interaction simulations. Finite Elem Anal Des 47:593-599

57. Long CC, Marsden AL, Bazilevs Y (2013) Fluid-structure interaction simulation of pulsatile ventricular assist devices. Comput Mech 52:971-981. https://doi.org/10.1007/s00466-013-0858-3

58. Long CC, Esmaily-Moghadam M, Marsden AL, Bazilevs Y (2014) Computation of residence time in the simulation of pulsatile ventricular assist devices. Comput Mech 54:911-919. https://doi.org/10.1007/s00466-013-0931-y

59. Long CC, Marsden AL, Bazilevs Y (2014) Shape optimization of pulsatile ventricular assist devices using FSI to minimize thrombotic risk. Comput Mech 54:921-932. https://doi.org/10.1007/ s00466-013-0967-z

60. Hsu M-C, Kamensky D, Bazilevs Y, Sacks MS, Hughes TJR (2014) Fluid-structure interaction analysis of bioprosthetic heart valves: significance of arterial wall deformation. Comput Mech 54:1055-1071. https://doi.org/10.1007/s00466-014-1059-4

61. Hsu M-C, Kamensky D, Xu F, Kiendl J, Wang C, Wu MCH, Mineroff J, Reali A, Bazilevs Y, Sacks MS (2015) Dynamic and fluid-structure interaction simulations of bioprosthetic heart valves using parametric design with T-splines and Fung-type material models. Comput Mech 55:1211-1225. https://doi.org/ 10.1007/s00466-015-1166-x

62. Kamensky D, Hsu M-C, Schillinger D, Evans JA, Aggarwal A, Bazilevs Y, Sacks MS, Hughes TJR (2015) An immersogeometric variational framework for fluid-structure interaction: application to bioprosthetic heart valves. Comput Methods Appl Mech Eng 284:1005-1053

63. Akkerman I, Bazilevs Y, Benson DJ, Farthing MW, Kees CE (2012) Free-surface flow and fluid-object interaction modeling with emphasis on ship hydrodynamics. J Appl Mech 79:010905

64. Akkerman I, Dunaway J, Kvandal J, Spinks J, Bazilevs Y (2012) Toward free-surface modeling of planing vessels: simulation of the Fridsma hull using ALE-VMS. Comput Mech 50:719-727

65. Wang C, Wu MCH, Xu F, Hsu M-C, Bazilevs Y (2017) Modeling of a hydraulic arresting gear using fluid-structure interaction and isogeometric analysis. Comput Fluids 142:3-14. https://doi.org/ 10.1016/j.compfluid.2015.12.004 
66. Wu MCH, Kamensky D, Wang C, Herrema AJ, Xu F, Pigazzini MS, Verma A, Marsden AL, Bazilevs Y, Hsu M-C (2017) Optimizing fluid-structure interaction systems with immersogeometric analysis and surrogate modeling: application to a hydraulic arresting gear. Comput Methods Appl Mech Eng 316:668-693

67. Yan J, Deng X, Korobenko A, Bazilevs Y (2017) Free-surface flow modeling and simulation of horizontal-axis tidal-stream turbines. Comput Fluids 158:157-166. https://doi.org/10.1016/ j.compfluid.2016.06.016

68. Castorrini A, Corsini A, Rispoli F, Takizawa K, Tezduyar TE (2019) A stabilized ALE method for computational fluidstructure interaction analysis of passive morphing in turbomachinery. Math Models Methods Appl Sci 29:967-994. https://doi. org/10.1142/S0218202519410057

69. Augier B, Yan J, Korobenko A, Czarnowski J, Ketterman G, Bazilevs Y (2015) Experimental and numerical FSI study of compliant hydrofoils. Comput Mech 55:1079-1090. https://doi.org/ 10.1007/s00466-014-1090-5

70. Yan J, Augier B, Korobenko A, Czarnowski J, Ketterman G, Bazilevs Y (2016) FSI modeling of a propulsion system based on compliant hydrofoils in a tandem configuration. Comput Fluids 141:201-211. https://doi.org/10.1016/j.compfluid.2015.07.013

71. Helgedagsrud TA, Bazilevs Y, Mathisen KM, Oiseth OA (2019) Computational and experimental investigation of free vibration and flutter of bridge decks. Comput Mech, Published online, https://doi.org/10.1007/s00466-018-1587-4

72. Helgedagsrud TA, Bazilevs Y, Korobenko A, Mathisen KM, Oiseth OA (2019) Using ALE-VMS to compute aerodynamic derivatives of bridge sections. Comput Fluids, Published online, https://doi.org/10.1016/j.compfluid.2018.04.037

73. Helgedagsrud TA, Akkerman I, Bazilevs Y, Mathisen KM, Oiseth OA, Isogeometric modeling and experimental investigation of moving-domain bridge aerodynamics. ASCE J Eng Mech, Accepted for publication

74. Kamensky D, Evans JA, Hsu M-C, Bazilevs Y (2017) Projectionbased stabilization of interface Lagrange multipliers in immersogeometric fluid-thin structure interaction analysis, with application to heart valve modeling. Comput Math Appl 74:2068-2088. https://doi.org/10.1016/j.camwa.2017.07.006

75. Yu Y, Kamensky D, Hsu M-C, Lu XY, Bazilevs Y, Hughes TJR (2018) Error estimates for projection-based dynamic augmented Lagrangian boundary condition enforcement, with application to fluid-structure interaction. Math Models Methods Appl Sci 28:2457-2509. https://doi.org/10.1142/S0218202518500537

76. Tezduyar TE, Takizawa K, Moorman C, Wright S, Christopher J (2010) Space-time finite element computation of complex fluidstructure interactions. Int J Numer Methods Fluids 64:1201-1218. https://doi.org/10.1002/fld.2221

77. Yan J, Korobenko A, Tejada-Martinez AE, Golshan R, Bazilevs Y (2017) A new variational multiscale formulation for stratified incompressible turbulent flows. Comput Fluids 158:150-156. https://doi.org/10.1016/j.compfluid.2016.12.004

78. van Opstal TM, Yan J, Coley C, Evans JA, Kvamsdal T, Bazilevs Y (2017) Isogeometric divergence-conforming variational multiscale formulation of incompressible turbulent flows. Comput Methods Appl Mech Eng 316:859-879. https://doi.org/10.1016/ j.cma.2016.10.015

79. Xu F, Moutsanidis G, Kamensky D, Hsu M-C, Murugan M, Ghoshal A, Bazilevs Y (2017) Compressible flows on moving domains: stabilized methods, weakly enforced essential boundary conditions, sliding interfaces, and application to gas-turbine modeling. Comput Fluids 158:201-220. https://doi.org/10.1016/ j.compfluid.2017.02.006

80. Tezduyar TE, Takizawa K (2019) Space-time computations in practical engineering applications: a summary of the 25 -year his- tory. Comput Mech 63:747-753. https://doi.org/10.1007/s00466018-1620-7

81. Takizawa K, Tezduyar TE (2012) Computational methods for parachute fluid-structure interactions. Arch Comput Methods Eng 19:125-169. https://doi.org/10.1007/s11831-012-9070-4

82. Takizawa K, Fritze M, Montes D, Spielman T, Tezduyar TE (2012) Fluid-structure interaction modeling of ringsail parachutes with disreefing and modified geometric porosity. Comput Mech 50:835-854. https://doi.org/10.1007/s00466-012-0761-3

83. Takizawa K, Tezduyar TE, Boben J, Kostov N, Boswell C, Buscher A (2013) Fluid-structure interaction modeling of clusters of spacecraft parachutes with modified geometric porosity. Comput Mech 52:1351-1364. https://doi.org/10.1007/s00466013-0880-5

84. Takizawa K, Tezduyar TE, Boswell C, Tsutsui Y, Montel K (2015) Special methods for aerodynamic-moment calculations from parachute FSI modeling. Comput Mech 55:1059-1069. https://doi.org/10.1007/s00466-014-1074-5

85. Takizawa K, Montes D, Fritze M, McIntyre S, Boben J, Tezduyar TE (2013) Methods for FSI modeling of spacecraft parachute dynamics and cover separation. Math Models Methods Appl Sci 23:307-338. https://doi.org/10.1142/S0218202513400058

86. Takizawa K, Tezduyar TE, Boswell C, Kolesar R, Montel K (2014) FSI modeling of the reefed stages and disreefing of the Orion spacecraft parachutes. Comput Mech 54:1203-1220. https://doi. org/10.1007/s00466-014-1052-y

87. Takizawa K, Tezduyar TE, Kolesar R, Boswell C, Kanai T, Montel K (2014) Multiscale methods for gore curvature calculations from FSI modeling of spacecraft parachutes. Comput Mech 54:14611476. https://doi.org/10.1007/s00466-014-1069-2

88. Takizawa K, Tezduyar TE, Kolesar R (2015) FSI modeling of the Orion spacecraft drogue parachutes. Comput Mech 55:11671179. https://doi.org/10.1007/s00466-014-1108-z

89. Takizawa K, Henicke B, Tezduyar TE, Hsu M-C, Bazilevs Y (2011) Stabilized space-time computation of wind-turbine rotor aerodynamics. Comput Mech 48:333-344. https://doi.org/10. 1007/s00466-011-0589-2

90. Takizawa K, Henicke B, Montes D, Tezduyar TE, Hsu MC, Bazilevs Y (2011) Numerical-performance studies for the stabilized space-time computation of wind-turbine rotor aerodynamics. Comput Mech 48:647-657. https://doi.org/10.1007/ s00466-011-0614-5

91. Takizawa K, Tezduyar TE, McIntyre S, Kostov N, Kolesar R, Habluetzel C (2014) Space-time VMS computation of windturbine rotor and tower aerodynamics. Comput Mech 53:1-15. https://doi.org/10.1007/s00466-013-0888-x

92. Takizawa K, Bazilevs Y, Tezduyar TE, Hsu M-C, Øiseth O, Mathisen KM, Kostov N, McIntyre S (2014) Engineering analysis and design with ALE-VMS and space-time methods. Arch Comput Methods Eng 21:481-508. https://doi.org/10.1007/s11831014-9113-0

93. Takizawa K (2014) Computational engineering analysis with the new-generation space-time methods. Comput Mech 54:193-211. https://doi.org/10.1007/s00466-014-0999-z

94. Takizawa K, Henicke B, Puntel A, Kostov N, Tezduyar TE (2012) Space-time techniques for computational aerodynamics modeling of flapping wings of an actual locust. Comput Mech 50:743-760. https://doi.org/10.1007/s00466-012-0759-x

95. Takizawa K, Henicke B, Puntel A, Kostov N, Tezduyar TE (2013) Computer modeling techniques for flapping-wing aerodynamics of a locust. Comput Fluids 85:125-134. https://doi.org/10.1016/ j.compfluid.2012.11.008

96. Takizawa K, Kostov N, Puntel A, Henicke B, Tezduyar TE (2012) Space-time computational analysis of bio-inspired flapping-wing aerodynamics of a micro aerial vehicle. Comput Mech 50:761778. https://doi.org/10.1007/s00466-012-0758-y 
97. Takizawa K, Tezduyar TE, Kostov N (2014) Sequentially-coupled space-time FSI analysis of bio-inspired flapping-wing aerodynamics of an MAV. Comput Mech 54:213-233. https://doi.org/ 10.1007/s00466-014-0980-x

98. Takizawa K, Tezduyar TE, Buscher A (2015) Space-time computational analysis of MAV flapping-wing aerodynamics with wing clapping. Comput Mech 55:1131-1141. https://doi.org/10.1007/ s00466-014-1095-0

99. Takizawa K, Bazilevs Y, Tezduyar TE, Long CC, Marsden AL, Schjodt K (2014) ST and ALE-VMS methods for patientspecific cardiovascular fluid mechanics modeling. Math Models Methods Appl Sci 24:2437-2486. https://doi.org/10.1142/ S0218202514500250

100. Takizawa K, Schjodt K, Puntel A, Kostov N, Tezduyar TE (2012) Patient-specific computer modeling of blood flow in cerebral arteries with aneurysm and stent. Comput Mech 50:675-686. https://doi.org/10.1007/s00466-012-0760-4

101. Takizawa K, Schjodt K, Puntel A, Kostov N, Tezduyar TE (2013) Patient-specific computational analysis of the influence of a stent on the unsteady flow in cerebral aneurysms. Comput Mech 51:1061-1073. https://doi.org/10.1007/s00466-012-0790$\mathrm{y}$

102. Suito H, Takizawa K, Huynh VQH, Sze D, Ueda T (2014) FSI analysis of the blood flow and geometrical characteristics in the thoracic aorta. Comput Mech 54:1035-1045. https://doi.org/10. 1007/s00466-014-1017-1

103. Suito H, Takizawa K, Huynh VQH, Sze D, Ueda T, Tezduyar TE (2016) A geometrical-characteristics study in patient-specific FSI analysis of blood flow in the thoracic aorta. In: Bazilevs Y, Takizawa K (eds) Advances in computational fluid-structure interaction and flow simulation: new methods and challenging computations, modeling and simulation in science, engineering and technology. Springer, Berlin, pp 379-386. https://doi.org/10. 1007/978-3-319-40827-9_29 ISBN 978-3-319-40825-5

104. Takizawa K, Tezduyar TE, Uchikawa H, Terahara T, Sasaki T, Shiozaki K, Yoshida A, Komiya K, Inoue G (2018) Aorta flow analysis and heart valve flow and structure analysis. In: Tezduyar TE (ed) Frontiers in computational fluid-structure interaction and flow simulation: research from lead investigators under forty 2018, modeling and simulation in science, engineering and technology. Springer, Berlin, pp 29-89. https://doi.org/10.1007/9783-319-96469-0_2 ISBN 978-3-319-96468-3

105. Takizawa K, Tezduyar TE, Uchikawa H, Terahara T, Sasaki T, Yoshida A (2019) Mesh refinement influence and cardiaccycle flow periodicity in aorta flow analysis with isogeometric discretization. Comput Fluids 179:790-798. https://doi.org/10. 1016/j.compfluid.2018.05.025

106. Takizawa K, Tezduyar K (2016) New directions in spacetime computational methods. In: Bazilevs Y, Takizawa K (eds) Advances in computational fluid-structure interaction and flow simulation: new methods and challenging computations, modeling and simulation in science, engineering and technology. Springer, Berlin, pp 159-178. https://doi.org/10.1007/978-3319-40827-9_13 ISBN 978-3-319-40825-5

107. Takizawa K, Tezduyar TE, Terahara T, Sasaki T (2018) Heart valve flow computation with the space-time slip interface topology change (ST-SI-TC) method and isogeometric analysis (IGA). In: Wriggers P, Lenarz T (eds) Biomedical technology: modeling, experiments and simulation. lecture notes in applied and computational mechanics. Springer, Berlin, pp 77-99. https://doi.org/ 10.1007/978-3-319-59548-1_6 ISBN 978-3-319-59547-4

108. Takizawa K, Montes D, McIntyre S, Tezduyar TE (2013) Spacetime VMS methods for modeling of incompressible flows at high Reynolds numbers. Math Models Methods Appl Sci 23:223-248. https://doi.org/10.1142/s0218202513400022
109. Takizawa K, Tezduyar TE, Hattori H (2017) Computational analysis of flow-driven string dynamics in turbomachinery. Comput Fluids 142:109-117. https://doi.org/10.1016/j.compfluid.2016. 02.019

110. Komiya K, Kanai T, Otoguro Y, Kaneko M, Hirota K, Zhang Y, Takizawa K, Tezduyar TE, Nohmi M, Tsuneda T, Kawai M, Isono M (2019) Computational analysis of flow-driven string dynamics in a pump and residence time calculation. In: IOP conference series earth and environmental science, vol 240, p 062014. https:// doi.org/10.1088/1755-1315/240/6/062014

111. Kanai T, Takizawa K, Tezduyar TE, Komiya K, Kaneko M, Hirota K, Nohmi M, Tsuneda T, Kawai M, Isono M (2019) Methods for computation of flow-driven string dynamics in a pump and residence time. Math Models Methods Appl Sci 29:839-870. https:// doi.org/10.1142/S021820251941001X

112. Otoguro Y, Takizawa K, Tezduyar TE (2017) Space-time VMS computational flow analysis with isogeometric discretization and a general-purpose NURBS mesh generation method. Comput Fluids 158:189-200. https://doi.org/10.1016/j.compfluid.2017. 04.017

113. Otoguro Y, Takizawa K, Tezduyar TE (2018) A general-purpose NURBS mesh generation method for complex geometries. In: Tezduyar TE (ed) Frontiers in computational fluid-structure interaction and flow simulation: research from lead investigators under forty-2018, modeling and simulation in science, engineering and technology. Springer, Berlin, pp 399-434. https://doi.org/10. 1007/978-3-319-96469-0_10 ISBN 978-3-319-96468-3

114. Otoguro Y, Takizawa K, Tezduyar TE, Nagaoka K, Mei S (2019) Turbocharger turbine and exhaust manifold flow computation with the space-time variational multiscale method and isogeometric analysis. Comput Fluids 179:764-776. https://doi.org/10.1016/j. compfluid.2018.05.019

115. Takizawa K, Tezduyar TE, Asada S, Kuraishi T (2016) Spacetime method for flow computations with slip interfaces and topology changes (ST-SI-TC). Comput Fluids 141:124-134. https:// doi.org/10.1016/j.compfluid.2016.05.006

116. Kuraishi T, Takizawa K, Tezduyar TE (2018) Space-time computational analysis of tire aerodynamics with actual geometry, road contact and tire deformation. In: Tezduyar TE (ed) Frontiers in computational fluid-structure interaction and flow simulation: research from lead investigators under forty-2018, modeling and simulation in science, engineering and technology. Springer, Berlin, pp 337-376. https://doi.org/10.1007/978-3-319-964690_8 ISBN 978-3-319-96468-3

117. Takizawa K, Tezduyar TE, Terahara T (2016) Ram-air parachute structural and fluid mechanics computations with the space-time isogeometric analysis (ST-IGA). Comput Fluids 141:191-200. https://doi.org/10.1016/j.compfluid.2016.05.027

118. Takizawa K, Tezduyar TE, Kanai T (2017) Porosity models and computational methods for compressible-flow aerodynamics of parachutes with geometric porosity. Math Models Methods Appl Sci 27:771-806. https://doi.org/10.1142/S0218202517500166

119. Kanai T, Takizawa K, Tezduyar TE, Tanaka T, Hartmann A (2019) Compressible-flow geometric-porosity modeling and spacecraft parachute computation with isogeometric discretization. Comput Mech 63:301-321. https://doi.org/10.1007/s00466-018-1595-4

120. Tezduyar TE, Ganjoo DK (1986) Petrov-Galerkin formulations with weighting functions dependent upon spatial and temporal discretization: applications to transient convection-diffusion problems. Comput Methods Appl Mech Eng 59:49-71. https:// doi.org/10.1016/0045-7825(86)90023-X

121. Le Beau GJ, Ray SE, Aliabadi SK, Tezduyar TE (1993) SUPG finite element computation of compressible flows with the entropy and conservation variables formulations. Comput Methods Appl Mech Eng 104:397-422. https://doi.org/10.1016/00457825(93)90033-T 
122. Tezduyar TE, Senga M (2006) Stabilization and shock-capturing parameters in SUPG formulation of compressible flows. Comput Methods Appl Mech Eng 195:1621-1632. https://doi.org/10. 1016/j.cma.2005.05.032

123. Corsini A, Menichini C, Rispoli F, Santoriello A, Tezduyar TE (2009) A multiscale finite element formulation with discontinuity capturing for turbulence models with dominant reactionlike terms. J Appl Mech 76:021211. https://doi.org/10.1115/1.3062967

124. Rispoli F, Saavedra R, Menichini F, Tezduyar TE (2009) Computation of inviscid supersonic flows around cylinders and spheres with the V-SGS stabilization and YZ $\beta$ shock-capturing. J Appl Mech 76:021209. https://doi.org/10.1115/1.3057496

125. Corsini A, Iossa C, Rispoli F, Tezduyar TE (2010) A DRD finite element formulation for computing turbulent reacting flows in gas turbine combustors. Comput Mech 46:159-167. https://doi.org/ 10.1007/s00466-009-0441-0

126. Hsu M-C, Bazilevs Y, Calo VM, Tezduyar TE, Hughes TJR (2010) Improving stability of stabilized and multiscale formulations in flow simulations at small time steps. Comput Methods Appl Mech Eng 199:828-840. https://doi.org/10.1016/j.cma.2009.06.019

127. Corsini A, Rispoli F, Tezduyar TE (2011) Stabilized finite element computation of NOx emission in aero-engine combustors. Int $\mathrm{J}$ Numer Methods Fluids 65:254-270. https://doi.org/10.1002/fld. 2451

128. Corsini A, Rispoli F, Tezduyar TE (2012) Computer modeling of wave-energy air turbines with the SUPG/PSPG formulation and discontinuity-capturing technique. J Appl Mech 79:010910. https://doi.org/10.1115/1.4005060

129. Corsini A, Rispoli F, Sheard AG, Tezduyar TE (2012) Computational analysis of noise reduction devices in axial fans with stabilized finite element formulations. Comput Mech 50:695-705. https://doi.org/10.1007/s00466-012-0789-4

130. Kler PA, Dalcin LD, Paz RR, Tezduyar TE (2013) SUPG and discontinuity-capturing methods for coupled fluid mechanics and electrochemical transport problems. Comput Mech 51:171-185. https://doi.org/10.1007/s00466-012-0712-z

131. Corsini A, Rispoli F, Sheard AG, Takizawa K, Tezduyar TE, Venturini P (2014) A variational multiscale method for particle-cloud tracking in turbomachinery flows. Comput Mech 54:1191-1202. https://doi.org/10.1007/s00466-014-1050-0

132. Rispoli F, Delibra G, Venturini P, Corsini A, Saavedra R, Tezduyar TE (2015) Particle tracking and particle-shock interaction in compressible-flow computations with the V-SGS stabilization and $\mathrm{YZ} \beta$ shock-capturing. Comput Mech 55:1201-1209. https:// doi.org/10.1007/s00466-015-1160-3

133. Cardillo L, Corsini A, Delibra G, Rispoli F, Tezduyar TE (2016) Flow analysis of a wave-energy air turbine with the SUPG/PSPG stabilization and discontinuity-capturing directional dissipation. Comput Fluids 141:184-190. https://doi.org/ 10.1016/j.compfluid.2016.07.011

134. Castorrini A, Corsini A, Rispoli F, Venturini P, Takizawa K, Tezduyar TE (2016) Computational analysis of wind-turbine blade rain erosion. Comput Fluids 141:175-183. https://doi.org/10. 1016/j.compfluid.2016.08.013
135. Takizawa K, Tezduyar TE, Otoguro Y (2018) Stabilization and discontinuity-capturing parameters for space-time flow computations with finite element and isogeometric discretizations. Comput Mech 62:1169-1186. https://doi.org/10.1007/s00466-018-1557$\mathrm{x}$

136. Castorrini A, Corsini A, Rispoli F, Venturini P, Takizawa K, Tezduyar TE (2019) Computational analysis of performance deterioration of a wind turbine blade strip subjected to environmental erosion. Comput Mech, published online, https://doi.org/ 10.1007/s00466-019-01697-0

137. Tezduyar TE, Aliabadi SK, Behr M, Mittal S (1994) Massively parallel finite element simulation of compressible and incompressible flows. Comput Methods Appl Mech Eng 119:157-177. https://doi.org/10.1016/0045-7825(94)00082-4

138. Takizawa K, Tezduyar TE (2014) Space-time computation techniques with continuous representation in time (ST-C). Comput Mech 53:91-99. https://doi.org/10.1007/s00466-013-0895-y

139. Takizawa K, Tezduyar TE, Sasaki T (2018) Estimation of elementbased zero-stress state in arterial FSI computations with isogeometric wall discretization. In: Wriggers P, Lenarz T (eds) Biomedical technology: modeling, experiments and simulation. Lecture notes in applied and computational mechanics. Springer, Berlin, pp 101-122. https://doi.org/10.1007/978-3-319-595481_7 ISBN 978-3-319-59547-4

140. Takizawa K, Tezduyar TE, Sasaki T (2017) Aorta modeling with the element-based zero-stress state and isogeometric discretization. Comput Mech 59:265-280. https://doi.org/10.1007/s00466016-1344-5

141. Sasaki T, Takizawa K, Tezduyar TE (2019) Aorta zero-stress state modeling with T-spline discretization. Comput Mech 63:13151331. https://doi.org/10.1007/s00466-018-1651-0

142. Sasaki T, Takizawa K, Tezduyar TE (2019) Medical-image-based aorta modeling with zero-stress-state estimation. Comput Mech, published online, https://doi.org/10.1007/s00466-019-01669-4

143. Takizawa K, Tezduyar TE, Sasaki T (2019) Isogeometric hyperelastic shell analysis with out-of-plane deformation mapping. Comput Mech 63:681-700. https://doi.org/10.1007/s00466-0181616-3

144. Saad Y, Schultz M (1986) GMRES: a generalized minimal residual algorithm for solving nonsymmetric linear systems. SIAM J Sci Stat Comput 7:856-869

Publisher's Note Springer Nature remains neutral with regard to jurisdictional claims in published maps and institutional affiliations. 